\title{
An Algorithmic Approach to Concept Exploration in a Large Knowledge Network (Automatic Thesaurus Consultation): Symbolic Branch-and-Bound Search vs. Connectionist Hopfield Net Activation
}

\author{
H. Chen and T. Ng \\ MIS Department, College of Business and Public Administration, University of Arizona, Tucson, AZ 85721. \\ E-mail: hchen@bpa.arizona.edu; tng@bpa.arizona.edu
}

\begin{abstract}
This paper presents a framework for knowledge discovery and concept exploration. In order to enhance the concept exploration capability of knowledge-based systems and to alleviate the limitations of the manual browsing approach, we have developed two spreading activation-based algorithms for concept exploration in large, heterogeneous networks of concepts (e.g., multiple thesauri). One algorithm, which is based on the symbolic Al paradigm, performs a conventional branch-and-bound search on a semantic net representation to identify other highly relevant concepts (a serial, optimal search process). The second algorithm, which is based on the neural network approach, executes the Hopfield net parallel relaxation and convergence process to identify "convergent" concepts for some initial queries (a parallel, heuristic search process). Both algorithms can be adopted for automatic, multiple-thesauri consultation. We tested these two algorithms on a large text-based knowledge network of about 13,000 nodes (terms) and 80,000 directed links in the area of computing technologies. This knowledge network was created from two external thesauri and one automatically generated thesaurus. We conducted experiments to compare the behaviors and performances of the two algorithms with the hypertext-like browsing process. Our experiment revealed that manual browsing achieved higher-term recall but lower-term precision in comparison to the algorithmic systems. However, it was also a much more laborious and cognitively demanding process. In document retrieval, there were no statistically significant differences in document recall and precision between the algorithms and the manual browsing process. In light of the effort required by the manual browsing process, our proposed algorithmic approach presents a viable option for efficiently traversing largescale, multiple thesauri (knowledge network).
\end{abstract}

\section{Introduction}

Knowledge discovery in databases that is based on statistical analysis, machine learning, and neural net com-

Received October 6. 1993; revised August 23, 1994; accepted September 8,1994 .

(C) 1995 John Wiley \& Sons, Inc. puting has attracted attention from researchers from several disciplines. In recent years, specialized software, powerful workstations, and even massively parallel computers have been used to perform extensive knowledge discovery on real-life databases. Advancement in hardware technology and the continuous development of practical, multiple-disciplined, "intelligent" analysis techniques has made "knowledge discovery" a highly promising area for information systems research and practice in the next decade.

With the computation power of prevailing hardware and the "intelligence" of many practical algorithms, knowledge discovery has also made possible the development of large knowledge bases. Knowledge discovery algorithms can explore and identify the underlying patterns in large databases and create much larger knowledge bases than it is possible to develop using manual, labor-intensive knowledge elicitation (Parsaye, Chignell, Khashafian, \& Wong 1989). The resulting discovered knowledge can also be consolidated and used in conjunction with other existing knowledge sources (either manually created or extracted from other sources). The surge in knowledge base development and rapid increases in the size of knowledge bases have prompted researchers to suggest knowledge management systems as a counterpart to database management systems (Kaufman, Michalski, \& Kerschberg 1991). The amount and diversity of discovered knowledge have called for the development of high-level, efficient knowledgc management tools.

A knowledge network may consist of knowledge discovered from real-life databases and knowledge extracted from existing domain-specific knowlcdge sources. In this paper we present our research concerning algorithmic concept exploration in a large network of knowledge. We propose two spreading activation-based 
algorithms for concept exploration. One is based on a conventional, serial branch-and-bound search algorithm and the other on neural net parallel relaxation. These algorithms can traverse (explore) a knowledge network automatically and suggest to users a set of concepts most relevant to their applications. This automatic concept exploration component can help alleviate the cognitive demand often associated with the manual browsing process and make the large-scale output of knowledge discovery methods more accessible and useful to users.

In Section 2 we first present a framcwork for knowledge discovery and concept exploration. The entities involved and their relationships are depicted in this framework. Section 3 presents an overview of issues related to concept exploration in a large knowledge network. We then discuss an implementation of concept exploration in a large text-based knowledge network in Section 4. Section 5 outlines a branch-and-bound spreading activation process and a Hopfield net-based parallel relaxation algorithm for concept exploration. A rationale for selecting these two methods will also be presented. We report the results of experimental evaluation of the two algorithms in Section 6 and conclude the paper in Section 7.

\section{A Framework for Knowledge Discovery and Concept Exploration}

Based on past research and our experience with various knowledge discovery methods, we developed a framework to depict the different entities involved in knowledge discovery and their intertwining relationships. The main entities involved in this framework include the underlying databases from which knowledge is acquired, knowledge discovery methods, the knowledge bases discovered by the algorithms and those imported from other domain-specific sources, and system-supported concept exploration or knowledge management tools for users. The definitions of databases and knowledge bases are sometimes blurred because of their historical roots in different disciplines such as artificial intelligence, database management systems, and information retrieval. In the context of our research, databases refer to online repositories of basic facts about objects and events in the world, e.g., employee files, transaction records, bibliographic records, etc. and knowledge bases are online repositories of high-level, abstract human knowledge represented in terms of heuristics, inferencing rules, problem-solving strategies, networks of interrelated concepts (concept space), and so on. These entities and their relationships are shown schematically in Figure 1 and discussed in the context of earlier research.

- Databases: As shown at the bottom of Figure 1, domain-specific databases that capture information or data of relevance to users' applications can be taken as major sources of knowledge. In the past few years, many major corporations and government agencies have used databases of drug side effects, retail shopping patterns, tax and welfare frauds, frequent flyer patterns, to identify application-specific knowledge. Frawley, Pietetsky-Shapiro, and Matheus (1991) present a gond overview of databases used in knowledge discovery.

The enormous sizes of real-life databases, which have frequently prevented human beings from conducting labor-intensive analysis, and the availability of unused computing cycles in many institutions have prompted the use of computers for knowledge discovery (Parsaye et al., 1989; Frawley et al., 1991). Massively parallel computers, and cven supcrcomputers, have also been considered for analysis of some really large business or scientific databases.

- Knowledge discovery methods: Various knowledge discovery techniques have been developed over the past few decades by statistics, information science, and artificial intelligence researchers.

Statistical algorithms typically examine quantitative data for the following purposes (Parsaye et al., 1989): clustering descriptors with common characteristics, e.g., factor analysis, principal components analysis (Morrison, 1976), and cluster analysis (Everitt, 1980); hypothesis testing for differences among different populations, e.g., $t$ test and analysis of variance (ANOVA) (Montgomery, 1976); trend analysis, e.g., time series analysis (Nelson, 1973; Morrison, 1976); and correlation between variables, e.g., correlation coefficient and linear/multiple regression analysis (Montgomery, 1976).

Recently, classical symbolic AI learning algorithms such as ID3 (Quinlan, 1983) and AQ (Michalski \& Larson, 1978) and resurgent neural net (NN) learning algorithms such as Backpropagation (Rumelhart, Hinton, \& Williams, 1986) have provided new perspectives for knowledge discovery. These techniques allow effective analysis of both qualitative and quantitative data. Unlike the statistical approach, which typically is based on some underlying models, assumptions, and stringent conditions, many AI-based techniques are more flexible, easier to use, more powerful, and produce output that is more meaningful to users. (For a complete overview of the AI-based learning techniques, readers are referred to Carbonell, Michalski, and Mitchell [1983], Dietterich and Michalski [1983], Knight [1990], and Frawley et al. [1991].

- Discovered knowledge and other knowledge sources: In addition to the mathematical formulas and parameters produced by statistical techniques, symbolic AIbased techniques produce outputs that are based on traditional knowledge representation schemes such as semantic net (SN), frame, decision trees, and logic (Parsaye, Chignell, Khoshafian, \& Wong, 1990; Quinlan, 1983; Michalski \& Stepp, 1983). Because most AIbased knowledge representations are grounded on cognitive research (human memory, problem-solving, story understanding, production systems) (Anderson, 1985), they are often considered more natural and understandable (for users) than statistical formulas or neural nets.

The amount of knowledge discovered by various knowledge discovery methods (e.g., number of rules 


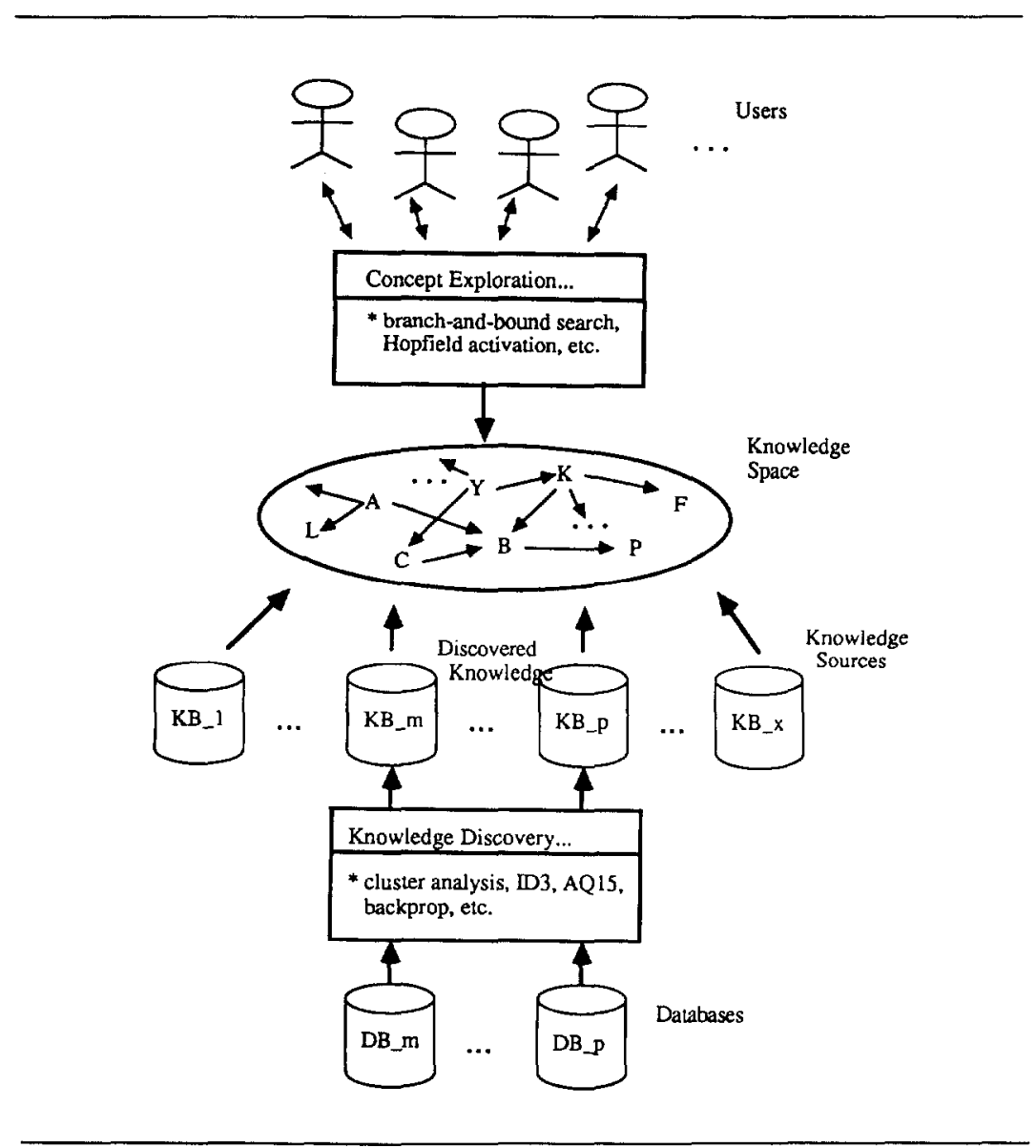

FIG. 1. A framework for knowledge discovery and concept exploration.

produced, number of nodes and links of a system-generated semantic net or neural net, levels and branches of a decision tree) sometimes may be substantial. For some applications, it may also be necessary to include domain-specific knowledge bases, expert systems, corporate rules and guidelines, data dictionaries, external thesauri, in an institution's completc knowlcdgc repository (shown in Figure 1 as the knowledge space).

- Concept exploration methods: In order to manage and utilize the different knowledge bases within a knowledge space, we believe it is necessary to develop some high-level, friendly, and efficient system-supported methods or tools for users. We refer to these methods and tools as the concept exploration methods in Figure 1. While knowledge discovery methods help generate knowledge bases from databases, concept exploration methods allow users to manage and utilize these knowledge bases effectively.

These concept exploration methods need to be highly interactive so that users can explore in the knowledge space freely and efficiently, articulate their conceptual models, and use whatever knowledge is relevant to their applications or tasks. While knowledge discovery methods are data driven as shown at the bottom of Figure 1, concept exploration needs to be user driven, as shown in the top part of Figure 1.
Kaufman et al. (1991) suggested a knowledge management component for knowledge discovery applications that can assist in the operation and use of knowledge bases and is similar to the database management function of commercial database management systems. They proposed counterparts of the relational database management system operators such as SELECT, PROJECT, JOIN, and INTERSECT for knowledge management. However, the proposed functionalities and roles for these operators remained vague and these authors have suggested further research investigating different high-level operators for different representation schemes. Our research has been aimed at examining in detail an "ACTIVATE" operator on semantic net and neural net representations that can assist in spreading activation-based inferencing.

\section{Spreading Activation in a Knowledge Network: Semantic Net or Neural Net?}

In this research, we focused on concept exploration methods in a large network structure of knowledge. We developed two spreading activation-based search meth- 
ods, one based on symbolic, serial branch-and-bound search and the other on the neural net parallel relaxation method. These two methods can be used, respectively, in a semantic net or neural net knowledge representation. The research represents a step toward developing a practical and useful knowledge management system.

Among the knowledge representation schemes frequently adopted in knowledge discovery research, network-based representation was often considered one of the most direct and natural representations. Within a network of interconnected nodes and directed links, the relationships among objects, knowledge, and patterns of interest can be represented explicitly. Two main paradigms of network representation need to be examined in detail in the context of our research: the symbolic AIbased semantic nets and the connectionist AI-based neural nets. While both representations continue to share some common bonds and exhibit some historical peculiarities, differences between them have become blurred due to the proliferation of network topologies and hybrid systems.

\section{Semantic Nets}

A semantic net is a structure for representing knowledge as a pattern of interconnected nodes and links (Sowa, 1991a). Modern semantic nets are often considered outgrowths of Quillian's (1968) work on semantic memory. Since then, many different versions have been implemented. Although the terminology and notations may vary, the following characteristics are common to most of them (Sowa, 1991a)

(1) Nodes represent concepts of entities, attributes, events, and states.

(2) Links represent conceptual relationships that hold between-concept nodes. Labels on the links specify relationship types.

Over the past two decades, many semantic net researchers have attempted to develop a formal theory and models of semantic nets. For example, it is possible to translate a semantic net into its equivalent first-order logic representation (Charniak, 1981). It has been shown that the class of semantic net languages cannot be differentiated from the class of nonsemantic net languages on the basis of representational adequacy (Shastri, 1991). More recently, some semantic net researchers have stressed the importance of developing efficient inferencing algorithms on semantic nets for real tasks. Bill Woods (Sowa, 1991b), one of the pioneers in semantic net research remarked:

A major gap, I believe, is the lack of sufficient emphasis on algorithmic uses of network representations to support various kinds of inference.
Many other researchers have also suggested developing efficient, real-time algorithms for inferencing, adopting semantic distance between two concepts as spreading activation heuristics, and measuring the performance of inferencing algorithms against real-life, large-scale applications (Sowa, 1991 b; Shastri, 1991).

The basis of most inferencing methods on semantic nets, however, is spreading activation, which is considered a variant of the state space traversal adopted in most symbolic Al-based systems (Winston, 1984; Rich \& Knight, 1991). Inference is performed by traversing (activating) the links and nodes connected to some initial nodes of concepts, with shorter paths considered preferable to longer ones, a characteristic of human reasoning (Anderson, 1985; Shastri, 1991). Conventional search techniques including depth-first-search (DFS), breadthfirst-search (BFS), branch-and-bound search, and $A^{*}$ search have often been used for state space traversal in applications such as the traveling salesman problem (Winston, 1984).

In this research, we adopted a branch-and-bound spreading activation algorithm for the following reasons. A branch-and-bound algorithm is considered an "optimal" search method, which aims at obtaining the shortest possible path during search. Identifying the shortest path in a large knowledge network is important because the objective of search is to find other very relevant concepts (i.e., neighboring concepts), instead of just any other concepts in the network. Therefore, $A^{*}$ and branch-and-bound were selected initially instead of the more popular (but nonoptimal) DFS or BFS method. A* search was later abandoned because of the lack of a proper underestimate measure in the concept exploration domain $\left(\mathrm{A}^{*}\right.$ terminates faster than branch-andbound due to its underestimate measure).

Branch-and-bound's systematic exploration of the neighboring structure of some initial nodes based on a priority queue made it suitable for the concept exploration application. Cohen and Kjeldsen's (1987) "constrained spreading activation" and Chen and Dhar's (1991) METACAT all incorporated branch-andbound's serial, optimal search property to some degree.

\section{Neural Nets}

Neural nets which represent knowledge, objects, and patterns in terms of interconnected nodes and weighted links have made an impressive come-back in recent years. There are several reasons for this, including the appearance of faster computers that can simulate larger networks and the development of new neural net architectures for real-life applications.

Neural nets are similar to semantic nets in terms of network representation. But distributed representations of neural nets (Hinton \& Sejnowski, 1986) do not use individual nodes to represent concepts or links to represent conceptual relationships. They use patterns of acti- 
vations over many units in the network. For example, a Hopfield net (Hopfield, 1982) may provide a distributed representation for a content-addressable memory in which each structure is stored as a collection of active units. Such special distributed representation allows the network to be more damage resistant, a property that exists in animal memory. In addition to the distributed representation property, neural nets perform parallel relaxation search, during which nodes are activated in parallel and are traversed until the network reaches a stable state. This process often is considered more efficient than serial, symbolic search because it makes use of states that have no analogues in symbolic search and because it maps naturally onto highly parallel hardware (Rich \& Knight, 1991).

The Hopfield net's parallel search on a single-layered network of nodes and weighted links and its convergence property made it suitable for automatic concept exploration. With the activation of some initial nodes, the Hopfield algorithm can activate their direct neighbors at the next iteration, combine and compute activation values from various sources, and continue the iterations until the activation strengths "die" out (a gradual damping process). In essence, the Hopfield net identifies other relevant concepts through a parallel and convergent approach. This neural network approach to search has only been used recently for information retrieval applications (Chen, Lynch, Basu, \& Ng, 1993) and its novel search capability makes it ideal for concept exploration. Other popular neural networks such as the Backpropagation networks or the Kohonen networks (Lippmann, 1987) were not considered for this research because of either inadequate network topology (i.e., both networks consist of multiple layers of objects) or inappropriate network activation algorithms (e.g., the Delta rule in Backpropagation is more suitable for learning). More details about the branch-and-bound and the Hopfield implementations adopted will be presented in Section 5 .

Many neural net and semantic net researchers consider localist representations of neural nets to be a variant of semantic nets (or conversely, semantic nets to be a variant of neural nets), in which each node and link, respectively, represents an individual concept and a conceptual relationship (Sowa, 1991b; Bechtel \& Abrahamsen, 1991; Rich \& Knight, 1991).

Systems developed by AI researchers frequently exhibit this resemblance to localist neural net and semantic net knowledge representations. For example, Anderson's (1983) ACT* nets and Fahlman's (1979) NETL both use nodes for concepts and allow some algorithmic spreading activation on the networks. Many hybrid systems developed in recent years employ symbolic and neural net characteristics. For example, Touretzky and Hinton (1988) and Gallant (1988) proposed connectionist production systems, and Derthick (1988) and Shastri (1991) developed different connectionist semantic networks.

In the research presented in this paper, we investi- gated a localist representation of a neural net which can also be perceived as a semantic net. To avoid confusion, we will use a more generic term, knowledge network, in the remainder of the paper. Our research examined the effects of implementing two different spreading activation methods: symbolic branch-and-bound versus connectionist parallel relaxation on a large hybrid neuralsemantic-net. Implementation issues such as computational efficiency, scalability of the algorithms for use in large-scale networks, and the performances of the two algorithms will be presented in detail.

\section{Concept Exploration in a Large Text-Based Knowledge Network: An Experiment}

We applied our proposed framework to a research environment where knowledge discovery and concept exploration were essential for information retrieval and intelligence analysis. The entities involved in this research environment consisted of many international computing researchers (users), a couple of document databases, and several knowledge sources. We have previously reported findings regarding automatic construction of networks of concepts for this application (Chen \& Lynch, 1991) and a blackboard design for integrating heterogeneous knowledge bases (Chen et al., 1993). We present here an overview of the application, emphasizing the concept exploration component in this environment.

\section{Databases}

The organization we studied is the Mosaic research group at the University of Arizona. Over the past decade group members have conducted research in the areas of foreign-nation studies and assessment of information technologies, focusing on the (former) Soviet Union and Eastern Europe (Russian/EE) (Goodman, Mehrer, Lynch, \& Roche, 1990; McHenry, Lynch, \& Snyder, 1990). Group members (analysts) collect articles and other forms of international computing-related academic publications, browse and study (foreign) documents collected, exchange ideas with foreign researchers via e-mail, telephone, and other means, visit foreign countries and organizations periodically, and attend major international conferences and professional meetings. They build their knowledge around certain subject areas, develop their own personal contacts with foreign researchers and organizations, and shape their beliefs, values, and judgment concerning international computing technologies and developments in specific countries of interest.

A significant portion of the Mosaic group memory and expertise has been captured by the Mosaic document databases. A custom-madc information storagc and retrieval system, built on top of INGRES, supports Mosaic research (Lynch, Snyder, McHenry, \& Vogel, 1990). The two databases considered most important to 
the Mosaic research environment both reside in the INGRES database management system. The "Russian" database, created manually, contains about 40,000 documents (article abstracts, newspaper articles, electronic mail exchanges, business cards) in a database of about 200 megabytes. In addition to this database, the Mosaic analysts also have extracted abstracts of recent computing-related articles from the DIALOG database, which they call the Public database. It consists of about 3,000 articles (20 megabytes). Some indexes had already been assigned to these documents by the DIALOG database. Because of operational concerns, this research only experimented with the Public database.

\section{Knowledge Discovery Methods}

In a previous paper (Chen \& Lynch, 1992), we reported detailed findings about automatic generation of knowledge bases from document databases. Several new cluster analysis algorithms were developed to produce knowledge bases (Saton, 1989; Everitt, 1980; Chen \& Lynch, 1992). These algorithms were based on the frequency of terms co-occurring in the documents and the resulting knowledge was captured in a semantic net representation where nodes represent different types of concepts and weighted links indicate their strengths of relevance.

In this paper, we use terms and indexes (terms used for indexing) interchangeably. When describing indexes and terms in the context of the semantic net or neural network representation, we also refer to them as nodes or concepts (to be consistent with the artificial intelligence terminology). We sketch below the procedure used for automatically creating knowledge bases. [Readers are referred to Chen and Lynch (1992) for details.]

(1) Determine unique indexes: We identified all indexes assigned to all the documents in the database [assigned previously by human indexers or generated automatically by automatic indexing techniques (Salton, 1989; Chen \& Lynch, 1992)].

(2) Weight computation: For each unique index, we computed its term co-occurrence probabilities with all other indexes based on the asymmetric "Cluster Function" developed by Chen and Lynch (1992). The term co-occurrence probability, which is a real number between 0 and 1 , indicates the "relevance" weight between any two indexes.

$$
\begin{aligned}
& W \operatorname{Ceight}\left(T_{j}, T_{k}\right)=\frac{\sum_{i=1}^{n} d_{i j k}}{\sum_{i=1}^{n} d_{i j}} \\
& \operatorname{Weight}\left(T_{k}, T_{j}\right)=\frac{\sum_{i=1}^{n} d_{i j k}}{\sum_{i=1}^{n} d_{i k}}
\end{aligned}
$$

They indicate the similarity weights from $T_{j}$ to $T_{k}$ (the first equation) and from $T_{k}$ to $T_{j}$ (the second equation). Where $d_{i j}$ indicates index $T_{j}$ in document $i$ (value: 0 or 1 ), $d_{i k}$ indicates index $T_{k}$ in document $i$ (value: 0 or 1), and $d_{i j k}$ indicates both indexes $T_{j}$ and $T_{k}$ are in document $i$ (value: 0 or 1 ).

The limitation of the popular symmetric co-occurrence coefficients, e.g., cosine, Dice, and Jaccard's, has been reported recently by Peat and Willett (1991). Their research showed that similar terms identified by symmetric co-occurrence functions tended to occur very frequently in the database that was being searched and thus did little or nothing to improve the discriminatory power of the original query. They concluded that this can help explain Sparck Jones' (1971) finding that the best retrieval results were obtained if only the less frequently occurring terms were clustered and if the more frequently occurring terms were left unclustered. We echo their observations and, in fact, we independently reached the same conclusion through our experience in developing several thesauri for capturing subject experts' domain concepts (in terms of keywords and relationships) for several applications (Chen \& Lynch, 1992; Chen, Schatz, Yim, \& Fye, in press). In Chen and Lynch (1992), we showed that the asymmetric function outperformed the cosine function in generating domain-specific thesaurus.

\section{Discovered Knowledge and Other Knowledge Sources}

We used the Public knowledge base for testing the concept exploration algorithms. The size and the subject area (general computing) of the Public knowledge base allowed us to implement and evaluate the algorithms on our hardware platform more easily. The Public knowledge base contains 1,488 concepts (nodes) and 44,496 weighted relationships (links). The weights associated with the links indicate the "strength" of relevance between two terms in the network. These weights are probabilities between 0 and 1 .

Use of a thesaurus or a knowledge base for "intelligent" information retrieval and management has been the focus of research in which many information science and computer science researchers have attempted to capture experts' domain knowledge for information retrieval or information management. For example, CoalSORT (Monarch \& Carbonell, 1987), a knowledgebased interface, facilitates the use of bibliographic databases in coal technology. A semantic net, representing an expert's domain knowledge, embodies the system's intelligence. GRANT, developed by Cohen and Kjeldsen (1987), is an expert system for finding sources of funding for given research proposals. Its search method-constrained spreading activation in a semantic net-makes inferences about the goals of the user and thus finds information not explicitly requested but likely to be useful. Chen and Dhar (1991) incorporated a portion of the Library of Congress Subject Headings (LCSH) into the design of an intelligent retrieval system. The system adopted a heuristics-based spreading activation algorithm to assist users in articulating their queries. The 
National Library of Medicine's Unified Medical Language System (UMLS) may be the largest-scale effort in integrating different knowledge sources (Humphreys \& Lindberg, 1989; McGray \& Hole, 1990; Lindberg \& Humphreys, 1990). It includes a Metathesaurus, a semantic network, and an information sources map. The Metathesaurus contains information about biomedical concepts and their representation in more than 10 different vocabularies and thesauri. The semantic network contains information about the types of terms (e.g., "discasc," "virus," etc.) in the Metathesaurus and the permissible relationships among these types. The information sources map contains information about the scope, location, vocabulary, and access conditions of biomedical databases of all kinds.

In an attempt to expand the knowledge coverage of our knowledge network, we included two more external knowledge sources (in the forms of thesauri) in our implementation: the complete ACM Computing Review Classification System (ACM CRCS) and a portion of the LCSH. The ACM CRCS represents the general computing categories used by the ACM for classifying computing literatures. The LCSH represents general computing terms selected by the Library of Congress for classifying computing-related books. Each knowledge source has its unique structure and vocabularies.

The ACM CRCS is based on a hierarchical structure. Four levels of specificity exist. Terms fan out level by level. Although its classification structure is simpler and its subjects are less specific, it does represent general computing terms and their relationships very nicely. We identified two types of terms from the ACM CRCS. The first type deals with specific topics which are similar to the LCSH, e.g., "information retrieval systems" and "machine learning." The second type of term, however, indicates general computing-related categories. These categories can be appended to terms in the ACM thesaurus. Examples of general categories are verification, documentation, testing. We identified 18 general categories and 1,141 specific terms from the ACM thesaurus. Five types of relationships were identified: BT/NT (broader/ narrower term) indicates hierarchical relationships between the specific terms, RT (related term) indicates an associative relationship (this relationship is shown in the parentheses following some terms), and ISA/INST (is-a and instance-of relationships) indicates the relationships between specific terms and general categories. For example, "Microprogram Design Aids-Verification" (a specific term) is-a kind of "Verification" (a general category) or conversely, an instance-of "Verification" is "Microprogram Design Aids-Verification." ISA and INST can be considered as special cases of the BT and NT relationships, respectively. We identified a total of 2,922 relationships from the $\Lambda$ CM CRCS.

The LCSH is network based and contains terms and cross-references between terms. Terms indicate topics. Five types of relationships exist between terms: USE/UF (use or used for) indicates a synonymous relationship, $\mathrm{RT}$ indicates an associative relationship, and BT/NT indicates hierarchical relationships. Our subset of the LCSH contains 10,972 terms and 32,702 relationships.

We present a summary of the structures of the three knowledge sources in terms of the frame-based representation in Figure 2. Collectively, they augment the knowledge of our system and they can be very useful in assisting searchers in articulating their queries and improving search recall. An analysis of the terms appearing in the three knowledge bases revealed that only a few hundred terms appeared in two or more knowledge sources (see Tablc 1). For cxample, Row 3, Columns 7 and 8 show that 122 terms in the Public Knowledge base also appearcd in the ACM CRCS, constituting $8.2 \%$ of the ACM CRCS terms. We can perceive the complete knowledge network as the union of three partially overlapping networks-the Public knowledge base, the ACM CRCS, and the LCSH.

\section{Concept Exploration Methods}

The rich semantics and cross-references provided in various knowledge bases enable users of such systems to get into a network of knowledge easily and to explore and navigate in this network. However, to perform query refinement and to identify relevant concepts efficiently and effortlessly in a large network/hierarchy of concepts (perhaps several thousand to a few million) and at the same time avoid both the classical hypertext "embedded digression problem" (a system can potentially confuse and disorient its user) and the "art museum phenomenon" (a system can cause users to spend a great of time while learning nothing specific) (Foss, 1989; Carmel, Crawford, \& Chen, 1992), requires an active and intelligent way to traverse multiple thesauri and multiple links. In the National Library of Medicine's UMLS (Humphreys \& Lindberg, 1989; McCray \& Hole, 1990; Lindberg \& Humphreys, 1990), which contains several million biomedical concepts and their relationships from more than 10 sources, browsing could become extremely cognitively demanding for users. System-aided concept exploration and multiple-thesauri consultation have become a pressing research issue.

"Spreading activation," a memory association mechanism that originated in human memory research, has been used successfully in various semantic net and neural net applications. Our application, which includes networks based on labeled links (the LCSH and the ACM CRCS) and weighted links (the Public knowledge base), is considered a hybrid system of semantic nets and neural nets.

In order to allow seamless spreading activation across all three knowlcdge bases, we developed a weight propagation scheme which assigned normalized weights to the labeled links (e.g., NT, RT, BT) based on the weights associated with the Public knowledge base. We can then 


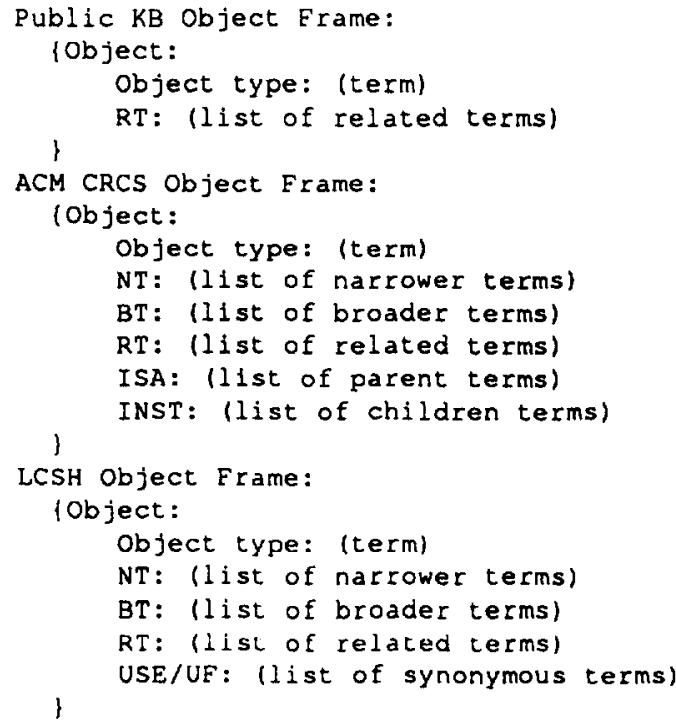

FIG. 2. Frame-hased representations for the knowledge sources.

traverse the resulting loosely coupled knowledge network by means of either symbolic state space search or neural net parallel relaxation. We outline the weight assignment and propagation scheme below:

(1) Elicit activation criteria: Two activation criteria need to be supplied by the user: Weights assigned to individual knowledge sources and weights assigned to different types of links. A scale of 0 to 10 for each knowledge source is used to indicate the searcher's preferred sources- 0 indicates the lowest preference level (i.e., the source is considered irrelevant) and 10 indicates the highest preference level. The ratings provided are used to determine the relative weights associated with different knowledge sources. Another $0-10$ scale is also used to elicit the user's preferences among three types of links: BT, NT, and RT. For example, assigning a higher rating to NT than to BT indicates the user's intention to traverse toward more specific concepts (through NT links). These ratings can be used by the spreading activation algorithms to determine the activation direction. By al- lowing users to indicate their preferred knowledge sources and link types, our activation algorithms can traverse the knowledge sources more efficiently and "intelligently." Setting these weights is a straightforward task for most users- the system prompts them to enter a numeric value for each parameter. The 0 10 scale allows them to indicate the relative importance of their preferences. Default values can also be used if a user chooses not to change the setting. (In our benchmark testing and user evaluation experiment, discussed in Section 6, default values for these weights were set as follows: Public/ACM/LCSH $=10 / 10 / 10$ and $\mathrm{RT} / \mathrm{NT} / \mathrm{BT}=3 / 10 / 1$.)

(2) Propagate connection weights: For the Public knowledge base, link weights have already been generated and stored. Because all three knowledge sources have some form of RT relationship, we can therefore use the RT link weights in the Public knowledge base as the basis for assigning weights to the RT links in the other two knowledge sources. The knowledge source and link type activation criteria obtained from the prior step can then be used to modify the assigned

TABLE 1. Terms/links in knowledge bases.

\begin{tabular}{|c|c|c|c|c|c|c|c|c|c|}
\hline \multirow[b]{2}{*}{ Terms in } & \multirow[b]{2}{*}{ Nodes } & \multirow[b]{2}{*}{ Links } & \multirow[b]{2}{*}{ Links/node } & \multicolumn{2}{|c|}{ Public } & \multicolumn{2}{|c|}{ ACM CRCS } & \multicolumn{2}{|c|}{$\mathrm{LCSH}$} \\
\hline & & & & Also in & $\%$ in & Also in & $\%$ in & Also in & $\%$ in \\
\hline Public & 1,488 & 44,486 & 29.9 & - & - & 122 & 8.2 & 177 & 11.9 \\
\hline ACM CRCS & 1,157 & 2,918 & 2.5 & 122 & 10.5 & - & - & 116 & 10.0 \\
\hline LCSH & 10,972 & 32,702 & 2.9 & 177 & 1.6 & 116 & 1.1 & - & - \\
\hline
\end{tabular}


weights to reflect the user's search criteria. For example, the NT link weights were computed as the product of the average RT weight in the Public knowledge base and the relative NT/RT weight solicited from users (i.e., $A R T * L W(N T) / L W(R T)$, as shown in the equations below). The same process was applied to the BT links. A sketch of the connection weight assignment for the different knowledge sources is shown below and the detailed spreading activation algorithms on this knowledge network are discussed in the next section.

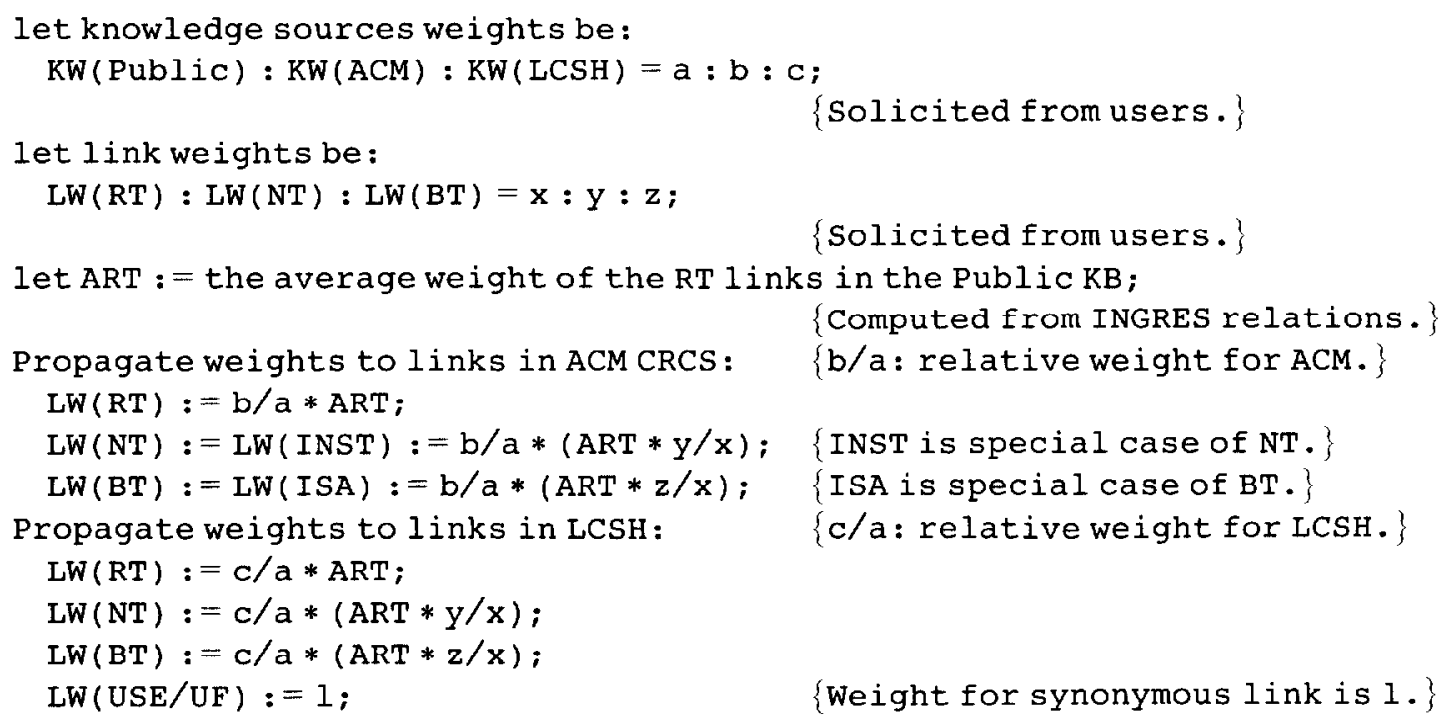

\{Weight for synonymous link is 1.$\}$

In conclusion, with multiple, heterogeneous thesauri, created either manually or automatically for experimental purposes, we were able to examine the feasibility of adopting spreading activation algorithms for "automatic thesaurus consultation." However, our proposed framework and algorithms were intended for the more general network-based knowledge-inferencing tasks.

\section{Two Algorithms for Spreading Activation}

In this section, we discuss in detail the two algorithms we developed for automatic and "intelligent" concept exploration in the knowledge network presented above. The novel features of the algorithms and a comparison of their behaviors are described.

\section{A Branch-and-Bound Spreading Activation Algorithm: Semantic Net Based}

Branch-and-bound search has been used frequently in statc space traversal for identifying optimal paths (Winston, 1984). Applications such as scheduling, network routing, and the traveling salesman problem typically adopt this search method. As explained earlier, branchand-bound was chosen over the more popular and yet simple DFS or BFS method because of its "optimal" search property, which was essential for finding the shortest paths and identifying a set of most relevant concepts in knowledge network. In Chen and Dhar (1991), we first reported the use of such a method for informa- tion retrieval. The algorithm automatically traversed an online thesaurus (the LCSH) and made term suggestions.

Our branch-and-bound implementation starts with terms provided by the user. These starting terms are assigned a value of $I$ as their node weights by the algorithm. (Our prototype system required an exact match between a search term and a node in the knowledge network. However, this can be improved in future development.) The terms are then used to activate their directly linked neighbors. Each activated neighbor receives a weight equal to the product of the weight of the activating node and the link weight. Based on the basic data structure adopted in branch-and-bound search, all activated nodes are put into a priority queue according to their associated weights. Terms with the heaviest weight in the queue are then used to activate their neighbors-terms which have equal weights are activated at the same time. Each node also records its starting term. Each activation is considered an iteration.

Branch-and-bound spreading activation repeats until a desirable user-defined state is reached (the stopping condition). When interacting with the algorithm adopted in our thesaurus consultation experiment, users are requested to provide a desired number of system-suggested associated terms, say $x$. This user-specified number is used to determine the stopping condition for the branch-and-bound iterations. The algorithm first computes the associated node weights generated from the first iteration and then uses the desired number of terms to determine a cut-off threshold. The cut-off helps obtain $x$ terms which are greater than the threshold. During the 
next iterations, the system performs the branch-andbound routine and combines weights of paths which originated from different starting terms. Nodes which obtain higher weights will thus be placed at the front of the priority queue.

In essence, our algorithm uses a user-specified number of terms to determine a stopping threshold for branch-and-bound. During iteration, it activates the highest-ranked nodes, computes node weights based on a simple multiplication function, and combines weights if the node can be reached from different starting terms. A more detailed sketch of the branch-and-bound spreading activation algorithm follows:

Assigning Weights to Symbolic Links. The initial status of the net is represented by the weighted links and nodes associated with the three thesauri as discussed earlier. $t_{i j}$ represents the weight from node $i$ to node $j$.

Initialization with User's Input. An initial set of starting terms $\left\{S_{1}, S_{2}, \ldots S_{m}\right\}$ is chosen by the user. Each node in the network (of $n$ nodes) which matches the starting terms is initialized to have a weight of 1 :

$$
\mu_{i}(0)=x_{i}, 0 \leq i \leq n-1
$$

$\mu_{i}(t)$ is the weight of node $i$ at iteration $t$. At time 0 , the nodes corresponding to the starting terms are assigned 1 .

The algorithm then creates a priority queue, $Q_{\text {priority }}$ based on the decreasing weights assigned to each node. Initially,

$$
Q_{\text {priority }}=\left\{S_{1}, S_{2}, \cdots S_{m}\right\}
$$

It also creates an output queue, $Q_{\text {oum }}$, to store the activated nodes during each iteration. Initially,

$$
Q_{\text {ouput }}=\{\}
$$

Activation, Weight Computation, and Iteration. During each iteration, the algorithm removes the highestweighted nodes in $Q_{\text {priority }}$, activates their neighboring nodes, and computes their neighbors' weights as follows:

$$
\mu_{j}(t+1)=\mu_{i}(t) \times t_{i j}
$$

As explained earlier, weight assignment is based on the product of the activating node weight and the link weight between the activating node and its neighbor. Recently activated nodes which had not been recorded earlier in $Q_{\text {ouput }}$ are inserted into the output queue, $Q_{\text {oupur }}$ (according to the order of their arrival). After computation, all expanded nodes in $Q_{\text {priority }}$ are resorted. In order to reward a node which can be reached by different starting terms (i.e., two different paths lead to the same node), the algorithm sums up its associated weights and assigns the result to the node. The algorithm then records this higher-weighted node in the priority queue. This heuristic of assigning higher weight to a node which can be reached from different starting nodes in the network has also been adopted in other spreading activation-based systems (Shoval, 1985; Cohen \& Kjeldsen, 1987; Chen \& Dhar, 1991).

Determining Stopping Condition. The algorithm solicits an expected number of system-suggested terms $(p)$ from the users. This number is used by the algorithm to determine the stopping condition for the branch-and-bound search. After the first iteration-all starting terms are activated because they have the same weights- $Q_{\text {prortity }}$ records the direct neighbors of all starting terms in decreasing weights. The algorithm identifies the pth node in $Q_{\text {priority }}$ and obtains its weight, $w_{p}$, as the threshold for stopping the branch-and-bound activation process (one of the stopping conditions). For most queries, $p$ terms were produced after the first iteration. However, occasionally multiple iterations were needed to obtain $p$ terms in $Q_{\text {priority. }}$. If the system was unable to produce $p$ terms after complete iteration (i.e., no more neighbors), the algorithm also terminated.

This user-specified threshold aimed to help generate the top $p$ terms relevant to the users' queries. During iteration, some terms which have higher weights than the terms in the queue will take up their positions in $Q_{\text {priority. }}$ The algorithm stops when the output queue, $Q_{\text {output }}$, consists of more than $p$ nodes (there may not be exactly $p$ nodes in the queue because the algorithm activates all highest-weighted nodes at the same iteration) or when the highest weight in $Q_{\text {priority }}$ is less than the user-defined threshold value, $w_{p}$, or when $Q_{\text {prioriy }}$ becomes null (i.e., all neighbors are exhausted).

The above branch-and-bound spreading activation algorithm is in essence a serial, optimal state space search process, during which "best" nodes get activated first. The user-supplied stopping condition allows the system to decide its exploration effort based on the users' expectation. These features compare favorably with those of other heuristics-based spreading activation systems (Cohen \& Kjeldsen, 1987; Shoval, 1985), which typically neither have a user-supplied threshold nor exhibit the branch-and-bound optimal search characteristics.

\section{A Hopfield Net Spreading Activation Algorithm: Neural Net Based}

The Hopfield net (Hopfield, 1982; Tank \& Hopfield, 1987), a classical method of inferencing in a single-layered, weighted network, presents an interesting and novel alternative to the serial state space traversal of the symbolic branch-and-bound algorithm. It performs a parallel relaxation search, during which nodes are activated in parallel and activation values from different sources are combined for each individual node. Neighboring nodes are traversed in order until the activation 
levels of nodes on the network gradually die out and the network reaches a stable state (convergence). As discussed earlier, a Hopfield net was chosen over other neural networks because of its parallel search and convergent properties and its single-layer topology (most other neural networks contain multiple layers of objects). A Hopfield net had been used successfully for various classification and optimization tasks (Lippmann, 1987; Simpson, 1990) and was also adopted recently in a blackboard-based retrieval system (Chen et al., 1993). Its search behavior in a large knowledge network, however, had not been examined in detail, especially in comparison with the more traditional serial search method.

A Hopfield net can be used as associated memory, where unknown input patterns (e.g., fuzzy queries) can be classified and disambiguated based on the knowledge embedded in the network. Our wcighted network of knowledge sources can be perceived as interconnections of neurons and synapses in the Hopfield net, where neurons represent concepts and synapses represent weighted links between pairs of concepts. Our implementation incorporates the basic Hopfield net iteration and convergence ideas. However, significant modification was also made to take into consideration the three different knowledge sources and other unique characteristics of our application.

Once the initial inputs and the weights have heen associated with the nodes in the knowledge sources, the algorithm activates neighboring terms, combines weighted links, performs a transformation function (a SIGMOID function, $f_{s}$ ), and determines the outputs of newly activated nodes. The process repeats until node outputs remain unchanged with further iterations. The node outputs then represent the concepts that best describe the initial search terms. A sketch of the Hopfield net activation algorithm follows:

Assigning Synaptic Weights. The "training" phase of the Hopfield net is completed when the weights have been propagated to all knowledge bases as discussed earlier. $t_{i j}$ represents the "synaptic" weight from node $i$ to node $j$.

Initialization with User's Input. An initial set of starting terms $\left\{S_{1}, S_{2}, \cdots S_{m}\right\}$ is chosen by a user and each node in the network that matches the starting terms is initialized to have a weight of 1 . Users also need to supply a desired number of suggested terms, $p$, as in the branchand-bound method.

$$
\mu_{i}(0)=x_{i}, 0 \leq i \leq n-1
$$

$\mu_{i}(t)$ is the output of node $i$ at time $t$ and $x_{i}$ which has a value between 0 and 1 , indicates the input pattern for node $i$. At time 0 , all input nodes are assigned 1 .

Activation, Weight Computation, and Iteration.

$$
\mu_{j}(t+1)=f_{s}\left[\sum_{i=0}^{n-1} t_{i j} \mu_{i}(t)\right], 0 \leq j \leq n-1
$$

where $f_{s}$ is the continuous SIGMOID transformation function as shown below (Knight, 1990; Dalton \& Deshmane, 1991)

$$
f_{s}\left(n e t_{j}\right)=\frac{1}{1+\exp \left[\frac{-\left(n e t_{j}-\theta_{j}\right)}{\theta_{0}}\right]}
$$

where net $_{j}=\sum_{i=0}^{n-1} t_{i j} \mu_{i}(t), \theta_{j}$ serves as a threshold or bias, and $\theta_{0}$ is used to modify the shape of the SIGMOID function.

This formula shows the parallel relaxation property of the Hopfield net. At each iteration, all nodes are activated at the same time. The weight computation scheme, $n e t_{j}=\sum_{i=0}^{n-1} t_{i j} \mu_{i}(t)$, is also a unique characteristic of the Hopfield net algorithm. Based on parallel activation, each newly activated node computes its new weight based on the summation of the products of the weights assigned to its neighbors and their synapses.

Convergence Stopping Condition. The above process is repeated until there is no change in terms of output between two iterations, which is accomplished by checking:

$$
\sum_{j=0}^{n-1}\left|\mu_{j}(t+1)-\mu_{j}(t)\right| \leq \epsilon
$$

where $\epsilon$ is the maximal allowable error (a small number). The final output represents the set of terms relevant to the starting term.

The algorithm presents the top $p$ terms among the final activated nodes if the number of final activated nodes is greater than $p$, the user's expected number of terms. If the number of final activated nodes is less than $p$, the system repeats the complete activation process by adopting a set of lower thresholds-lower $\theta_{0}$ and $\theta_{j}$ values-in order to derive more activated nodes. In our implementation, we allow the system to lower its thresholds three times, incrementally. If no more terms can be derived after lowering the thresholds three times, the algorithm terminates and presents the results from the last activation. From our experiments, it appears that these default thresholds were able to handle most user requests. Only rarely did the system need to lower its thresholds more than once or twice.

This threshold-tuning effort was critical to our application as it was in other Hopfield net applications (Lippmann, 1987; Knight, 1990). Our objective in tuning was to obtain a manageable number of branches at each iteration and a reasonable number of iterations. After experimentation, a default value of 0.11 for $\theta_{j}$ and a value of 0.05 for $\theta_{0}$ were selected as global default values for the network. The other three sets of $\left(\theta_{j}, \theta_{0}\right)$ were: $(0.065$, 
$0.047),(0.056,0.0464)$, and $(0.047,0.0458)$, respectively.

Both the branch-and-bound and the Hopfield net activation algorithms were developed in $\mathrm{C}$ and run on a DECStation 5000/120 (25 MIPS machine, ULTRIX, 1.2 gigabyte disk). The three knowledge sources were stored as three individual flat files in our testing.

\section{System Evaluation}

In order to examine the novel characteristics and performances of our two algorithms, we performed a benchmark testing and a user evaluation experiment, respectively. The aim of the benchmark testing was to reveal the computational characteristics of the two algorithms, specifically the number of iterations performed by each method, the computing times, and the "source of knowl- edge" for each system-suggested term. The user evaluation, on the other hand, aimed at addressing performance issues. In this section we first present sample system sessions based on the two algorithms. We then present our evaluation results.

\section{Sample Sessions}

Sample sessions of branch-and-bound and Hopfield net spreading activations are presented below. Comments are enclosed in parentheses. The subject was requested to identify topics (with the help of the system) relevant to "KIDS: A Query and Inference System Based upon Knowledge Indexed Deductive Search," by K. Lee, a Georgia State University Ph.D. dissertation, 139 pages, 1989. An abstract of this dissertation was also presented to the subject. (Details about the experiment will be discussed in the following subsection.)

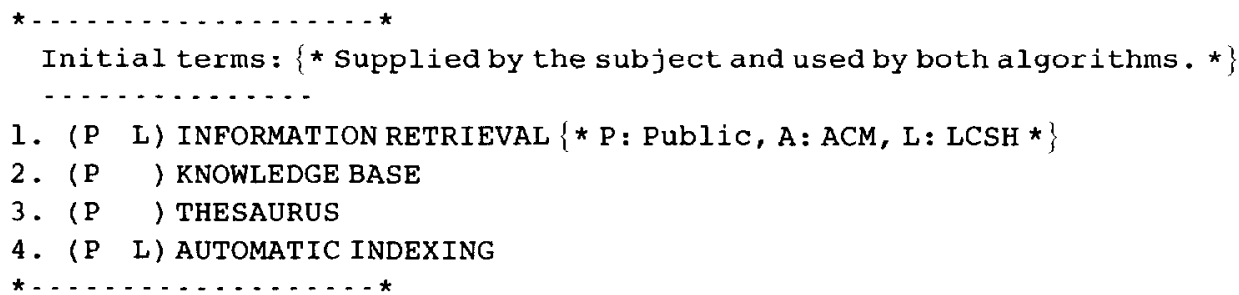

\section{A. Branch-and-Bound Activation.}

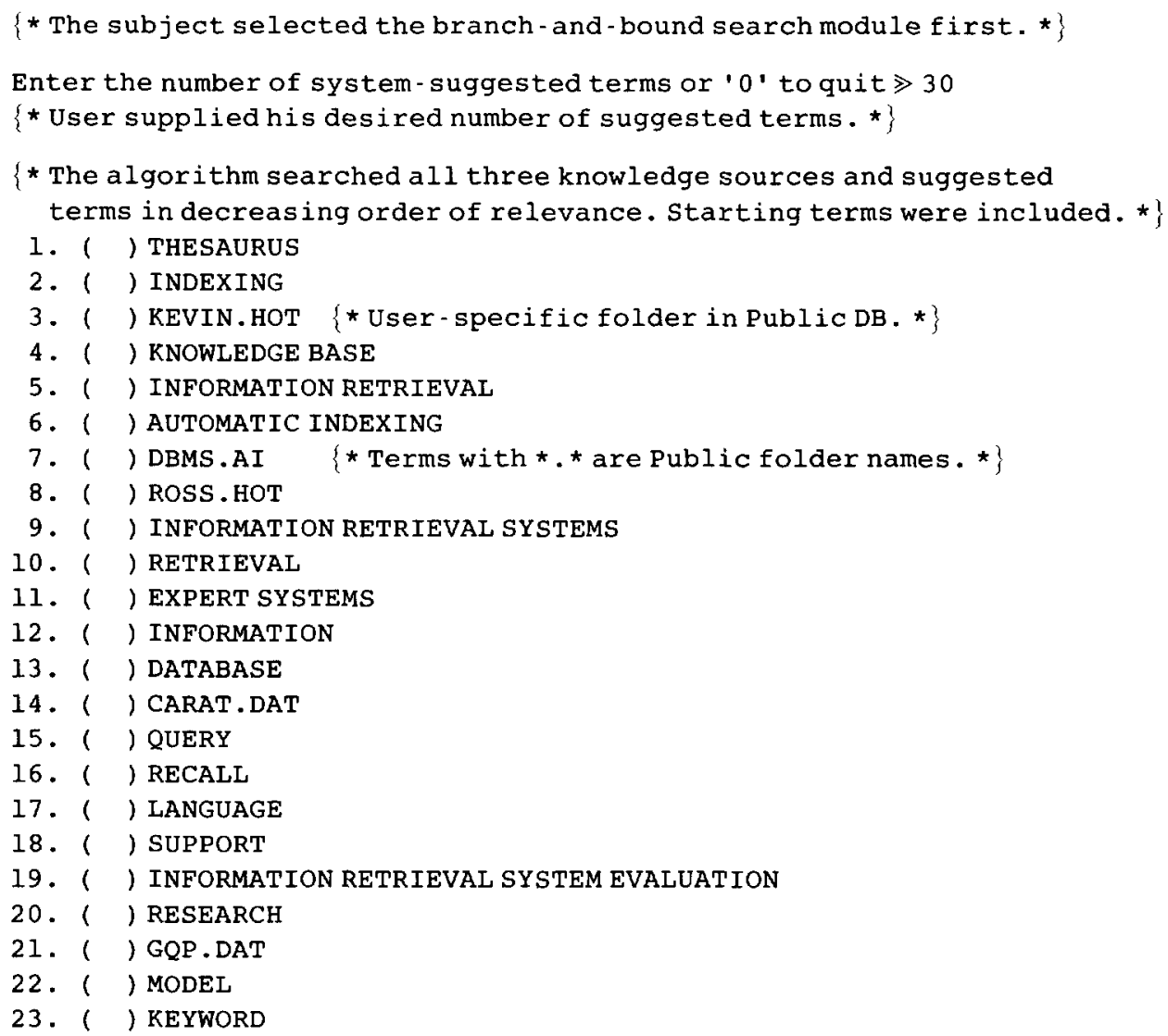


24. ( ) PRECISION

25. ( ) USER INTERFACES

26. ( ) PETER.HOT

27. ( ) ARTIFICIAL INTELLIGENCE

28. ( ) MANAGEMENT

29. ( ) EXPERT SYSTEM

30. ( ) LOGIC

31. ( ) OBJECT

32. ( ) SEMANTIC.MDL

33. ( ) DATABASE MANAGEMENT SYSTEMS

34. () EXPERT

35. ( ) DESIGN

Enter numbers [1 to 35] or ' 0 ' to quit: 1, 2,4-6, 9, 16, 19, 24

\{* The subject selected desired terms. *\}

$\{$ * The system 1 isted the user-selected terms and their sources . *

1. (P) THESAURUS

2. (P) INDEXING

3. (P) KNOWLEDGE BASE

4. (P L) INFORMATION RETRIEVAL

5. ( $P$ L) AUTOMATIC INDEXING

6. ( $P$ L) INFORMATION RETRIEVAL SYSTEMS

7. (P) RECALL

8. (P ) INFORMATION RETRIEVAL SYSTEM EVALUATION

9. (P) PRECISION

Enter the number of system-suggested terms of ' 0 ' to quit $\gg 50$

\{ The subject used the selected terms to activate the branch-and-bound algorithm again. *

$\{$ * More terms were suggested. *

1. ( ) KEVIN.HOT

2. ( ) INDEXING

3. ( ) INFORMATION RETRIEVAL

4. ( ) RECALL

........

54. ( ) DATA STRUCTURES

55. ( ) PERFORMANCE

56. ( ) QUERY.OPT

57. ( ) ARTIFICIAL INTELLIGENCE

58. ( ) KEYWORD

59. ( ) THESAURI

60. ( ) USER INTERFACES

$\{$ * More selections. * $\}$

Enter numbers [ 1 to 60 ] or ' 0 ' to quit: $2-6,8,10,11,14,15$

$\{*$ Notice that terms were ranked in di erent order than had been suggested previously. This was because of the different starting terms. *

1. (P) ) INDEXING

2. ( $P$ L) INFORMATION RETRIEVAL

3. (P) RECALL

4. (P) PRECISION

5. ( $P$ L) INFORMATION RETRIEVAL SYSTEMS

6. (P ) INFORMATION RETRIEVAL SYSTEM EVALUATION

7. (P) THESAURUS

8. ( $\mathrm{P}$ L) AUTOMATIC INDEXING

9. ( L) INFORMATION STORAGE AND RETRIEVAL SYSTEMS * A new term. *

10. ( $P$ ) KNOWLEDGE BASE

Enter the number of system-suggested terms or ' 0 ' to quit $\gg 0$

\{ * The subject decided to stop the search process. *\}

$\{*$ A total of 10 terms were selected. Six terms were suggested by the 
branch-and-bound algorithm and they came from two knowledge sources: the Public KB and the LCSH. *

\section{B. Hopfield Net Activation.}

\{ * The subject selected the Hop"eld net search module. similar interface was used for the Hop"eld net search process. *

Enter the number of system-suggested terms or ' 0 ' to quit $\gg 10$

$\{$ * Notice that the suggested terms were different from those suggested by the branch-and-bound algorithm. *

1. ( ) INDEXING

2. ( ) SELLING - INFORMATION STORAGE AND RETRIEVAL SYSTEMS

3. ( ) KEVIN. HOT

4. ( ) INFORMATION RETRIEVAL SYSTEM EVALUATION

5. () RECALL

6. () EXPERT SYSTEMS

7. ( ) THESAURUS

8. ( ) DBMS.AI

9. ( ) ROSS.HOT

10. ( ) INFORMATION STORAGE AND RETRIEVAL SYSTEMS

11. ( ) INFORMATION RETRIEVAL

12. ( ) KNOWLEDGE BASE

13. ( ) AUTOMATIC INDEXING

Enter numbers [ 1 to 13 ] or ' 0 ' to quit: $1,2,4,5,7,10-13$

1. (P) INDEXING

2. ( L) SELLING - INFORMATION STORAGE AND RETRIEVAL SYSTEMS

3. (P ) INFORMATION RETRIEVAL SYSTEM EVALUATION

4. (P) RECALL

5. (P) THESAURUS

6. ( L) INFORMATION STORAGE AND RETRIEVAL SYSTEMS

7. ( $P$ L) INFORMATION RETRIEVAL

8. (P ) KNOWLEDGE BASE

9. (P L) AUTOMATIC INDEXING

Enter the number of system-suggested terms or ' 0 ' to quit $\gg 30$ ........

Enter number [ 1 to 40 ] or ' 0 ' to quit: $3-7,9,33,35,36,38$ $\ldots \ldots \ldots$

Enter numbers [ 1 to 67 ] or ' 0 ' to quit: 0

\{* The system listed his final selections. *\}

1. (P) PRECISION

2. ( $P$ ) INFORMATION RETRIEVAL

3. (P) INDEXING

4. (P L) AUTOMATIC INDEXING

5. (P ) RECALL

6. ( L) AUTOMATIC ABSTRACTING \{* Suggested by HP, not BAB. *

7. (L) AUTOMATIC CLASSIFICATION \{ suggested by HP, not BAB . *\}

8. ( L) AUTOMATIC INFORMATION RETRIEVAL (* Suggested by HP, not BAB. *\}

9. (P ) INFORMATION RETRIEVAL SYSTEM EVALUATION

10. ( $P$ ) THESAURUS

11. ( L) INFORMATION STORAGE AND RETRIEVAL SYSTEMS

12. ( $\mathrm{P}$ ) KNOWLEDGE BASE

$\{$ * A total of 12 terms were selected. Eight terms were suggested by the Hop"eld net algorithm. Terms 6,7 , and 8 were di erent from those suggested by the branch - and - bound algorithm. * 


\section{Benchmark Testing}

We first performed a benchmark testing of the two algorithms using 30 sample queries (generated by the experimenters). Each query consisted of terms of various degrees of specificity (e.g., Artificial Intelligence vs. Natural Language Processing) and different numbers of search terms. We tested five cases each for queries with 1 term, 2 terms, 3 terms, 4 terms, 5 terms, and 10 terms, a total of 30 cases. A few examples of the queries used, all in the computing area, were: 1 term (Natural Language Processing), 2 terms (Group Decision Support Systems, Collaboration), 3 terms (Systems Analysis and Design, Simulation and Modeling, Optimization).

For each query, we selected terms from different knowledge sources, "P" for the Public knowledge base, "A" for the ACM CRCS, and "L" for the LCSH, as shown in Table 2. Some terms may have appeared in more than one knowledge source. The results shown in Table 2 reveal the number of iterations, the computing times, and the sources of knowledge for the query terms and the system-suggested terms. Note that the branchand-bound algorithm performed serial iteration, while the Hopfield net performed parallel relaxation at each iteration. The reason for investigating the source of knowledge for system-suggested terms was to show the extent to which each algorithm branched out and utilized knowledge from other knowledge sources.

In response time, the branch-and-bound algorithm clearly performed better than the Hopfield net parallel activation. A MINITAB two-sample $t$ test (Ryan, Joiner, \& Ryan, 1985) showed that on average the neural net took 24.5 seconds (standard deviation, $[S D]=8.34$ ) while the semantic net took 6.9 seconds $(S D=2.42)$. The difference was statistically significant (value of two-sample $t$ test, $T=11.10$ and significance level, $p=.0000$ ). This was clearly because the branch-and-bound search performed only a fixed number of serial explorations, while the Hopfield net searched a much larger search space during the parallel activation process.

Despite the variation in the number of starting terms, the response times for both methods increased only slightly when the number of starting terms was increased. This finding is important, especially when considering complex, fuzzy queries which often contain many starting terms (a scenario in which searchers need the most help from the system). The reason for this small

TABLE 2, Results of benchmark testing.

\begin{tabular}{|c|c|c|c|c|c|}
\hline Case & $\begin{array}{l}\text { No. of } \\
\text { terms }\end{array}$ & $\begin{array}{l}\text { Query terms in } \\
(P, A, L)\end{array}$ & $\begin{array}{c}\text { Suggested terms in } \\
\text { NN:(P,A,L)/SN:(P,A,L) }\end{array}$ & $\begin{array}{l}\text { No. of iterat. } \\
\text { NN/SN }\end{array}$ & $\begin{array}{c}\text { Times (secs) } \\
\text { NN/SN }\end{array}$ \\
\hline 1 & 1 & $(1,1,1)$ & $(12,7,7) /(5,7,2)$ & $18 / 12$ & $21 / 11$ \\
\hline 2 & 1 & $(1,0,1)$ & $(5,0,16) /(19,0,2)$ & $15 / 21$ & $14 / 8$ \\
\hline 3 & 1 & $(1,1,1)$ & $(11,5,11) /(15,0,0)$ & $14 / 16$ & $18 / 10$ \\
\hline 4 & 1 & $(0,0,1)$ & $(0,0,20) /(0,0,20)$ & $11 / 20$ & $10 / 11$ \\
\hline 5 & 1 & $(1,0,1)$ & $(4,4,19) /(16,0,3)$ & $17 / 20$ & $26 / 10$ \\
\hline 6 & 2 & $(2,1,0)$ & $(19,2,3) /(23,1,0)$ & $21 / 23$ & $18 / 6$ \\
\hline 7 & 2 & $(2,0,2)$ & $(16,0,8) /(18,0,1)$ & $19 / 23$ & $22 / 8$ \\
\hline 8 & 2 & $(2,0,0)$ & $(20,3,4) /(21,0,0)$ & $20 / 23$ & $24 / 5$ \\
\hline 9 & 2 & $(2,1,1)$ & $(11,5,11) /(19,0.0)$ & $15 / 23$ & $16 / 4$ \\
\hline 10 & 2 & $(2,1,2)$ & $(11,0,12) /(20,0,0)$ & $27 / 22$ & $29 / 4$ \\
\hline 11 & 3 & $(3,0,1)$ & $(20,0,18) /(18,0,0)$ & $19 / 22$ & $31 / 5$ \\
\hline 12 & 3 & $(1,2,1)$ & $(4,11,8) /(14,0,2)$ & $22 / 17$ & $34 / 6$ \\
\hline 13 & 3 & $(2,1,3)$ & $(22,1,8) /(15,0,2)$ & $18 / 19$ & $29 / 6$ \\
\hline 14 & 3 & $(1,3,1)$ & $(20.2,2) /(19.0 .0)$ & $16 / 22$ & $23 / 8$ \\
\hline 15 & 3 & $(1,2,2)$ & $(13,9,3) /(18,0.1)$ & $9 / 21$ & $10 / 4$ \\
\hline 16 & 4 & $(2,2,4)$ & $(17,4,4) /(16,1,1)$ & $17 / 20$ & $11 / 6$ \\
\hline 17 & 4 & $(3,2,2)$ & $(11,2,13) /(19,0,1)$ & $19 / 23$ & $31 / 5$ \\
\hline 18 & 4 & $(2,3,2)$ & $(18,5,6) /(17,0,2)$ & $24 / 21$ & $33 / 4$ \\
\hline 19 & 4 & $(1,3,4)$ & $(18,2,5) /(20,1,1)$ & $19 / 24$ & $32 / 7$ \\
\hline 20 & 4 & $(1,2,1)$ & $(15,8,3) /(12,2,1)$ & $18 / 22$ & $6 / 7$ \\
\hline 21 & 5 & $(1,4,1)$ & $(19,4,6) /(19,0,1)$ & $16 / 24$ & $27 / 3$ \\
\hline 22 & 5 & $(4,2,2)$ & $(10,1,12) /(19,0,1)$ & $15 / 19$ & $27 / 4$ \\
\hline 23 & 5 & $(3,2,4)$ & $(2,0,18) /(0,0,21)$ & $11 / 21$ & $23 / 9$ \\
\hline 24 & 5 & $(5,0,1)$ & $(19,0,3) /(17,0,1)$ & $23 / 17$ & $33 / 9$ \\
\hline 25 & 5 & $(5,0,1)$ & $(20,0,1) /(23,0,0)$ & $12 / 23$ & $30 / 12$ \\
\hline 26 & 10 & $(8,0,3)$ & $(11,0,13) /(12,0,19)$ & $17 / 39$ & $34 / 6$ \\
\hline 27 & 10 & $(10,1,3)$ & $(13,2,10) /(18,3,2)$ & $25 / 19$ & $32 / 7$ \\
\hline 28 & 10 & $(8,0,4)$ & $(16,0,8) /(19,0,2)$ & $24 / 21$ & $36 / 8$ \\
\hline 29 & 10 & $(9,1,5)$ & $(19,1,6) /(21,0,1)$ & $27 / 22$ & $25 / 9$ \\
\hline 30 & 10 & $(8,2,3)$ & $(20,2,3) /(20,0,2)$ & $28 / 21$ & $31 / 6$ \\
\hline Average & 5 & $(3.1,1.2,1.9)$ & $(14.5 .2 .5,8.5) /(16.4 .0 .5 .3 .0)$ & $18.8 / 21.3$ & $24.5 / 6.9$ \\
\hline
\end{tabular}


variation was that our branch-and-bound search decided a threshold based on the user's expected number of terms. The Hopfield net thresholds $\left(\theta_{0}\right.$ and $\left.\theta_{j}\right)$, on the other hand, were robust enough to guarantee a reasonable number of hits.

Knowledge sources activated by the branch-andbound algorithm appeared to be more strongly associated with the origins (knowledge sources) of the starting terms than those activated by the Hopfield net. For example, when using the branch-and-bound method, if the starting term was from the LCSH, then the final branchand-bound suggested terms were more likely to be from the LCSH than from other sources. The IIopfield net, on the other hand, appeared to invoke the different knowledge sources more evenly. As shown in Table 2, for most queries, the Hopfield net (NN) almost always produced terms from all three knowledge sources (i.e., more evenly), while the branch-and-bound (SN) often produced terms from only a couple of knowledge sources (usually identical to the sources of the query terms). We believe this was because the parallel relaxation process branched out to other knowledge sources more efficiently than the serial search, with the result that combining evidence from different activated nodes as implemented in Hopfield net activation caused more even activation of terms from all sources.

\section{User Evaluation}

The second user evaluation experiment was designed to reveal the "quality" of the algorithms' suggestions. We aimed to find out whether the two algorithms were able to help identify more relevant terms and documents and perform more efficiently than the conventional hypertext-like browsing method (i.e., manual thesaurus browsing). Term recall/precision, document recall/ precision, and time spent were used as performance measures. We also recorded the subjects' verbal comments on their retrieval operations. The Public database and three computing-related knowledge bases were used in the experiment.

We enlisted three subjects for this experiment, two of whom were advanced (3-4 year) Ph.D. students in an information systems department and one was a M.S. student in a library school. All were working on theses in the database, artificial intelligence, or information retrievalrelated areas. We selected for testing six dissertation abstracts which appeared in the 1991 spring issue of SIGIR Forum (a publication of the ACM special interest group on information retrieval). These dissertations were all in areas that were familiar to the subjects with titles such as "Retrieval by Similarity in a Knowledge Base of Reusable Code," "Cognitive Aspects of Human-Computer Interaction: Mental Models in Database Query Writing."

The subjects were shown the title and a half-page abstract for each dissertation. They were asked to read this information carefully and were requested to identify the subject areas or topics that they thought they would need to explore in order to develop a comprehensive overview and understanding of the dissertation. An experimenter recorded the subject-suggested terms.

After the initial term solicitation, subjects were asked to use one algorithm and then the other to help them find other topics that might supplement their initial sets of topics. The order of presentation of the two algorithms had previously been randomized for each task. The same experimenter recorded all the selected terms. The same menu-driven interface (as shown in the previous sample session) was used for both algorithms and was operated by the experimentcr. For cach task, the intcrfacc allowed each subject to pick terms suggested by the algorithms, use the newly picked terms to activate the algorithms, pick more terms, activate again, and so on until the subject decided to stop. After using both algorithms, subjects were asked to examine the lists of terms they initially presented and the terms they picked from the system-suggested lists and decide whether they were still relevant (sometimes terms may no longer be considered relevant after a subject has identified other more precise terms).

After using the algorithm-based interface, we asked subjects to browse the three knowledge bases for terms that might supplement their initial sets of terms (subjectsuggested terms). A simple hypertext-like interface was used to navigate through the three knowledge bases. For each task, each subject examined all three knowledge bases separately, in the subject's own choice of order. Each subject typed in one term at a time using a specific thesaurus. The subject then chose one relevant term (if any occurred) from the thesaurus-suggested list and repeated the same process. In essence, the hypertext-like browsing system only looked up terms which were directly linked to a search term in a chosen knowledge base. On the other hand, the two algorithms performed an optimal (or convergent), multiple-link, multiple-thesaurus search for relevant terms, in contrast to the often laborious manual browsing process that is widely used in library and bibliographic database retrieval settings (Chen \& Dhar, 1987). After browsing all knowledge bases, subjects were asked to re-examine the lists of selected terms and to confirm their selections.

After the above term selection process (both algorithmic and manual), we proceeded to a document selection and evaluation phase. Due to difficulty in operationalizing the recall and precision measures (especially recall) (Salton, Allan, \& Buckley, 1994), we adopted a document evaluation design similar to the one reported in Ekmekcioglu, Robertson, and Willett (1992). Subjects were asked to examine different sets of ranked documents for their relevance to the corresponding dissertation abstract. Four lists of terms, representing subject suggested, branch-and-bound suggested, Hopfield net suggested, and browsing selected terms, were used, respectively, to retrieve the 15 most pertinent abstracts from the Public database. Document ranking was based on the number 
of terms that were associated with each abstract. In addition, we also randomly generated 15 abstracts that did not match with any of the selected terms. Depending on the number of overlapping abstracts, the number of documents in each collection for each task ranged from 43 to 68 . By mixing relevant and irrelevant documents and soliciting subjects' evaluation, we were able to find out which set of terms was most helpful in suggesting relevant documents.

On average, each subject spent about 6.2 hours to complete all six tasks through the above stages: subject's term suggestion, two algorithm activations, manual browsing, and document evaluation. We also logged the complete interactions and recorded subjects' verbal comments. Two sets of performance analysis were conducted: One focused on terms and the other on documents. We report the results below.

Performance Analysis on Term Retrieval. We uscd the final lists of terms picked by each individual subject (i.e., subject-suggested, algorithm-suggested, and browsingsuggested terms) for each task as the target list of relevant terms. We then computed the term recall, term precision, time spent, contribution rate, and reviewing rate of the branch-and-bound suggested list (semantic net), the Hopfield net suggested list (neural net), and the manual browsing selected list (MB). Results are shown in Figure 3.

Interestingly, there was more agreement between subjects in their initial lists than their final lists. Most subjects used the terms in the SIGIR abstracts to start their searches, thus there was a significant overlap of terms used. However, after initiating the manual browsing process and the algorithms, their selections varied significantly. We believe this was because of the differences between the subjects' backgrounds and their interpretations of the SIGIR abstracts.

A. Term Recall. Term recall indicated the portion of the target list which was found on each of the four lists. Manual browsing resulted in a (statistically) significantly higher recall (at $p=10 \%$ level) over the branch-andbound (SN) and Hopfield net (NN) algorithms (SN vs. $\mathrm{NN}$ vs. MB, $p=.013$ ). Two two-sample $t$ tests confirmed this finding ( $\mathrm{SN}: \mathrm{MB}=0.34: 0.44, p=.021 ; \mathrm{NN}: \mathrm{MB}$ $=0.33: 0.44, p=.014$ ). Between the two algorithms, the branch-and-bound algorithm had a slightly higher recall than the Hopfield net algorithm, but the difference was not significant ( $\mathrm{SN}: \mathrm{NN}=0.34: 0.33, p=.778$ ). After extensive manual browsing, subjects were able to obtain a larger set of relevant terms (than resulted from using the algorithmic process).

B. Term Precision. Term precision indicated the portion of each list that appeared in the target list. In contrast to the recall results, manual browsing resultcd in a significantly lower precision value compared with the algorithm-based systems ( $\mathrm{SN}$ vs. NN vs. $\mathrm{MB}, p=.000$ ). This was mainly because the number of terms suggested via manual browsing was much larger than that of terms suggested by the two algorithms, and only a few were judged relevant by the subjects. Two two-sample $t$ tests also confirmed this finding ( $\mathrm{SN}: \mathrm{MB}=0.19: 0.02, p$ $=0.000 ; \mathrm{NN}: \mathrm{MB}=0.18: 0.02, p=.000)$. Although the branch-and-bound algorithm had a slightly higher precision than the Hopfield net algorithm, the difference was not significant ( $\mathrm{SN}: \mathrm{NN}=0.19: 0.18, p=.495)$.

C. Time Spent. Because the hypertext-like interface suggested many more terms than the two algorithms, the time spent on manual browsing was significantly longer than that spent on either of the two algorithms (SN vs. NN vs. MB, $p=.000$ ). On average, each subject spent 16.2 minutes browsing, while each spent 5.9-7.2 minutes using either algorithm for each task. Two two-sample $t$ tests revealed the same result (SN:MB $=5.9: 16.2, p$ $=.000 ; \mathrm{NN}: \mathrm{MB}=7.2: 16.2, p=.000)$. Between the two algorithmic systems, subjects spent less time using the branch-and-bound system, but the difference was not significant $(\mathrm{SN}: \mathrm{NN}=5.9: 7.2, p=.281$ ).

D. Contribution Rate. We defined a new measure, contribution rate, to indicate the number of terms picked by subjects per time unit ( 1 minute). ANOVA showed that the contribution rate of manual browsing was significantly lower than that of either algorithm (SN vs. NN vs. $\mathrm{MB}, p=.001)$. On average, the manual browsing process contributed 0.58 term per minute, while branch-andbound and Hopfield net systems contributed 1.44 and 1.22 terms per minute, respectively. Two two-sample $t$ tests also confirmed this finding ( $\mathrm{SN}: \mathrm{MB}=1.44: 0.58, p$ $=.000 ; \mathrm{NN}: \mathrm{MB}=1.22: 0.58, p=.003)$. However, the difference between the two algorithms' contribution rates was not significant ( $\mathrm{SN}: \mathrm{NN}=1.44: 1.22, p=.436$ ).

E. Reviewing Rate. A subject was expected to spend about the same amount of time reviewing terms suggested by algorithms and terms returned from manual browsing. Nonetheless, in this experiment, the reviewing rate (the number of terms reviewed per time unit-1 minute in this case) for manual browsing was significantly faster than that for both algorithms (SN vs. NN vs. MB, $p=.000$ ). Individual two-sample $t$ tests confirmed the result (SN:MB $=7.5: 29.6, p=.000 ; \mathrm{NN}: \mathrm{MB}$ $=6.9: 29.6, p=.000$ ). There was no significant difference between the two algorithms ( $\mathrm{SN}: \mathrm{NN}=7.5: 6.9, p=.565$ ). The hypertext-like browsing process was clearly more time pressed and cognitively demanding than the algorithmic process.

Using their own terms, subjects were able on average to achieve a $30 \%$ recall level. Their precision level was at $100 \%$ - all terms they initially supplied were judged relevant later on. Each algorithm was able to double the number of terms subjects selected, as shown by the $33 \%$ 


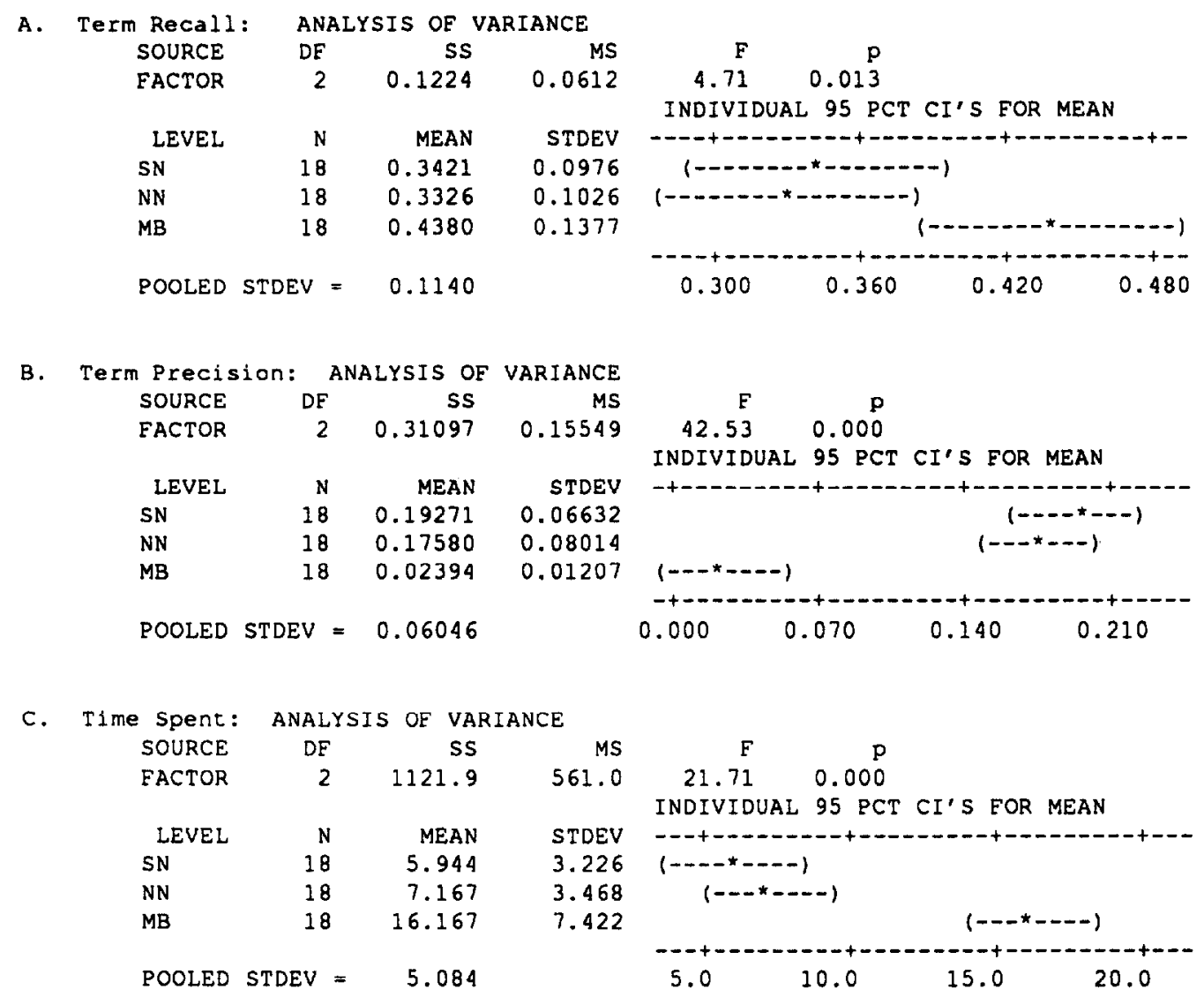

D. Contribution Rate: ANALYSIS OF VARIANCE

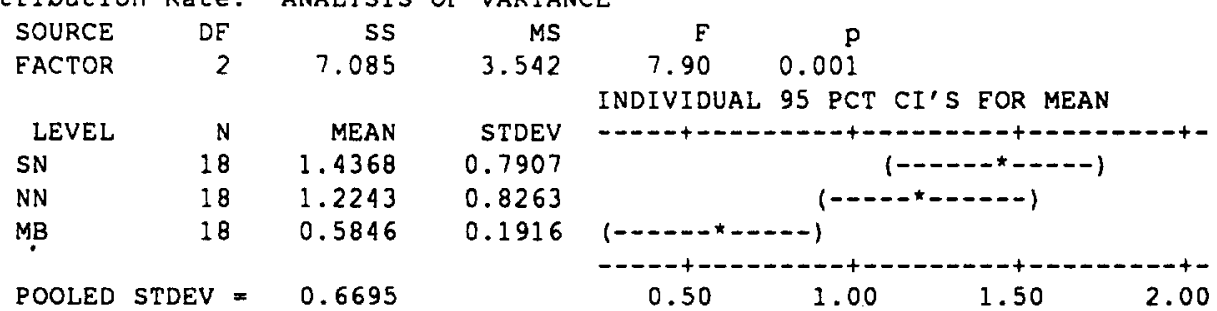

E. Reviewing Rate: ANALYSIS OF VARIANCE

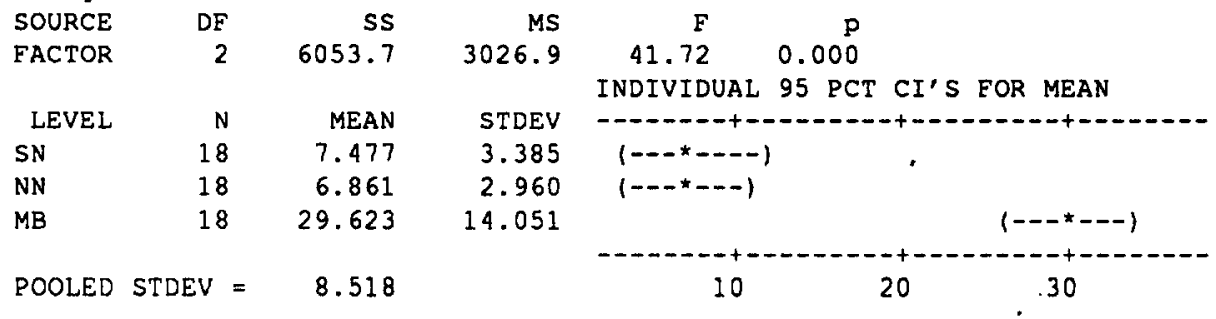

FIG. 3. Results of performance analysis on term retrieval.

and $34 \%$ recall values from the two algorithmic systems. The human's ability to recognize objects (much better than recall of objects) has been well documented in the cognitive psychology literature (Anderson, 1985; Chen \& Lynch, 1992). The algorithms' suggestions in effect served as an excellent memory-jogging tool for users dur- 
ing concept exploration. The same memory-jogging factor also enabled manual browsing to provide more terms $-44 \%$ recall value for manual browsing. However, the time spent and effort involved in manual browsing were enormous for all subjects.

An interesting observation from the experiment, based on the subjects' verbal protocols, was that the manual browsing process often resulted in serendipitous and/or off-the-track browsing behavior as reported in Carmel et al. (1992). A subject commented, "I just want to have a quick look at this term. It is related to what I am doing (subject's own research) right now. It's not relevant to this query." In addition, manual browsing provided a lot of opportunity for being distracted. While reviewing a list of terms, a subject said, "I am curious about this term. Let me see what it is." We observed that when subjects used the algorithms, they reviewed the suggested terms more slowly and treated them more seriously and carefully than when performing manual browsing. Subjects glanced or skimmed through most terms retrieved from manual browsing. Another observation was that subjects were easily frustrated when they reached the end of a particular link during the manual browsing process. A subject said, "It's a dead end. . . . It's a dead end, again!" Whereas the physical appearances of all three knowledge bases were left open for subjects to browse and examine, both of our algorithms hid physical appearance from subjects.

In conclusion, manual browsing achieved higherterm recall but lower-term precision than the algorithmic systems. It was also a much more laborious and cognitively demanding process.

Performance Analysis on Document Retrieval. For document retrieval performance analysis, we first compared documents obtained from four different sources: subjectsuggested (Subject), branch-and-bound algorithm (SN), Hopfield net algorithm (NN), and manual browsing process (MB; Figure 4A, B). We then combined the two sets of algorithm-related documents to determine performance differences among human effort (Subject), algorithmic approach ( $\mathrm{SN} / \mathrm{NN}$ ), and manual browsing approach (MB; Figure 4C, D). (The two algorithms suggested different sets of documents which can be combined easily in a system.) Document recall and precision results are discussed below.

A. Document Recall (Subject, SN, NN, MB). Document recall indicated the portion of the target document list which was found in each of the four lists. The document recall for manual browsing was somewhat higher than that for subject-suggested, branch-and-bound, and Hopfield net algorithms, but the overall ANOVA did not show a significant difference (Subject:SN:NN:MB $=0.31: 0.27: 0.29: 0.43, p=.202$ ). However, the two-sample $t$ test between the branch-and-bound procedure and the manual browsing process did indicate a significant difference (SN:MB $=0.27: 0.43, p=.068)$. Other individual two-sample $t$ tests did not show significant difference (Subject:SN, $p=.627$; Subject:NN, $p=.784$; Subject: $\mathrm{MB}, p=.180 ; \mathrm{SN}: \mathrm{NN}, p=.851 ; \mathrm{NN}: \mathrm{MB}, p=.116$ ).

B. Document Precision (Subject, SN, NN, MB). Document precision indicated the portion of each document list that appeared in the target document list. The document precision values for subject-suggested and manual browsing sets appeared to be higher than those of the others. However, there was no statistically significant difference among four sources (Subject:SN:NN:MB $=0.21: 0.15: 0.14: 0.19, p=.583$ ). Individual two-sample $t$ tests also did not reveal any significant differences (Subject:SN, $p=.334$; Subject:NN, $p=.275$; Subject:MB, $p$ $=.735 ; \mathrm{SN}: \mathrm{NN}, p=.938 ; \mathrm{SN}: \mathrm{MB}, p=.441 ; \mathrm{NN}: \mathrm{MB}, p$ $=.356$ ). (Because the terms provided by one subject for a particular task did not retrieve any documents, the sample size for Subject was reduced from 18 to 17 as shown in Figure 4B, D.)

C. Document Recall (Subject, SN/NN, MB). The document recall values for the combined algorithms and manual browsing were almost the same. While these values were higher than for subjects, the difference was not statistically significant (Subject:SN/NN:MB $=0.31$ : 0.43:0.43, $p=.305$ ). Individual two-sample $t$ tests confirmed the same finding (Subject:SN/NN, $p=.167$; Subject:MB, $p=.180 ; \mathrm{SN} / \mathrm{NN}: \mathrm{MB}, p=.978$ ).

D. Document Precision (Subject, SN/NN, MB). The document precision for $\mathrm{SN} / \mathrm{NN}$ was less than that for Subject and MB. However, there was no significant difference among them (Subject:SN/NN:MB $=0.2 \mathrm{l}$ : $0.14: 0.19, p=.460$ ). Individual two-sample $t$ tests confirmed the result (Subject:SN/NN, $p=0.247$; Subject: $\mathrm{MB}, p=.735 ; \mathrm{SN} / \mathrm{NN}: \mathrm{MB}, p=.314)$.

In conclusion, we did not observe significant differences between the relevant documents suggested by the algorithms and those generated via the manual browsing process (in document recall and precision). However, the algorithmic thesaurus consultation approach and the manual thesaurus browsing process all could contribute to a larger set of relevant documents for users (than was discovered without such aids). In light of the effort required of the manual browsing process, our proposed algorithmic approach appeared to be a viable option for efficiently traversing large-scale, multiple thesauri (knowledge networks).

\section{Conclusion}

This paper presents a framework for knowledge discovery and concept exploration in a large knowledge network. The potentially large amount of knowledge that can be discovered by various $\Lambda \mathrm{I}$, statistical, and neural net learning algorithms and the need to integrate differ- 

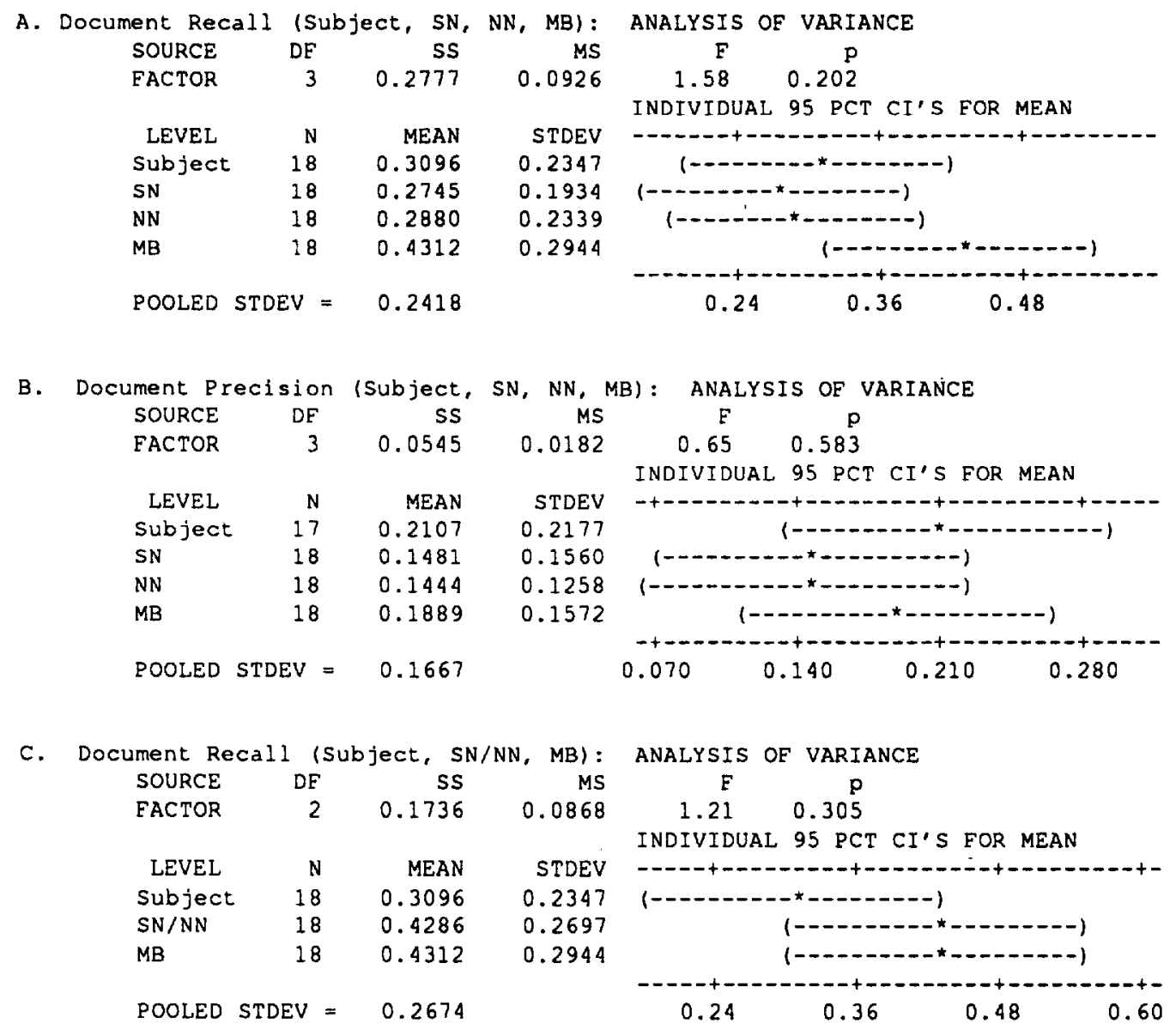
D. Document Precision (Subject, SN/NN, MB): ANALYSIS OF VARIANCE

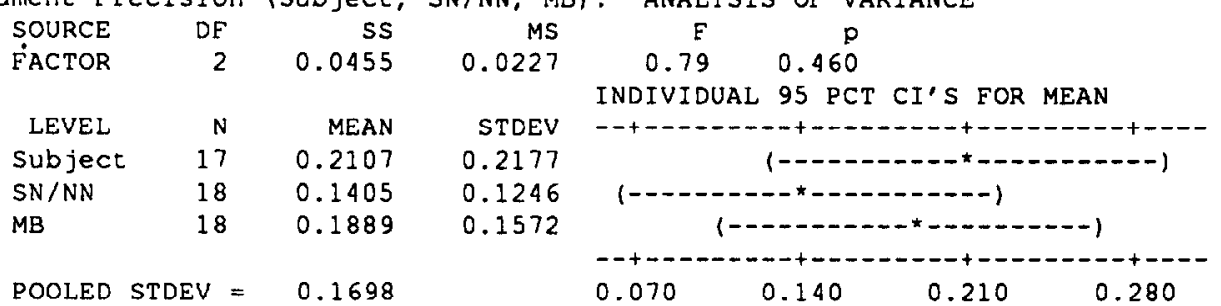

FIG. 4. Results of performance analysis on document retrieval.

ent sources of knowledge have made knowledge management or concept exploration in a large knowledge space an important area for research.

Based on a hybrid network represcntation of a semantic net and a neural net, we have proposed two paradigms for automatic traversal in heterogeneous knowledge networks: One based on a symbolic, branch-and-bound search algorithm and the other on a Hopfield net parallel relaxation algorithm. The branch-and-bound search tried to find the "best" search path based on some cost computations and a system-maintained priority queue of incomplete paths. The Hopfield net activation, on the other hand, performed parallel activation of neighboring nodes and combined weights from all neighbors until the network reached a convergent state. Our design goal was to permit obtaining real-time system performance in large-scale knowledge networks.

We tested these two algorithms in an application where three knowledge sources were used for information retrieval. One knowledge source was created by cluster analysis algorithms and the other two knowledge bases were extracted from external sources, all having 
network structure. The complete knowledge network consisted of 13,617 nodes and 80,106 links. The two algorithms and the knowledge sources were implemented on a DECStation $5000 / 120$.

Two experiments were conducted in an attempt to reveal the performance levels of the two methods and their novel characteristics in comparison to the conventional hypertext-like browsing process. The benchmark testing used 30 sample 1-term, 2-term, 3-term, 4-term, 5-term, and 10-term queries to determine the computation times and the activation patterns of the various knowledge sources using the two algorithms. The user evaluation experiment allowed three graduate student subjects to interact with the two algorithms for six tasks of concept cxploration and document retrieval.

In the benchmark testing, the branch-and-bound algorithm was faster than the Hopfield net activation. The average branch-and-bound search took about $6.9 \mathrm{sec}$ onds and the Hopfield net activation took 24.5 seconds. However, the parallel relaxation process of the Hopfield net appeared to have helped activate multiple thesauri better than the serial branch-and-bound search, regardless of the source of the initial concepts.

The user evaluation experiment revealed that manual browsing achieved higher-term recall but lower-term precision in comparison to the algorithmic systems. However, it was also a much more laborious and cognitively demanding process. In document retrieval, there were no statistically significant differences in document recall and precision between the algorithms and the manual browsing process. In light of the effort required to accomplish the manual browsing process, our proposed algorithmic approach appeared to be a viable option for efficiently traversing large-scale, multiple thesauri (knowledge network).

In conclusion, we believe this research has provided insights concerning development of robust and "intelligent" network-based knowledge management and inferencing systems. A sample application of the proposed design could be for "automatic thesaurus consultation" of multiple, heterogeneous thesauri, created either manually or automatically. An algorithmic approach to concept exploration in a large knowledge network can be performed under a real-time computation constraint and could be very useful for interactive, large-scale document retrieval applications.

\section{Acknowledgments}

This project was supported mainly by a National Institutes of Health BRSG grant S07RR07002 and a National Science Foundation grant IRI-9211418. We would also like to thank K. Basu, K. Ng, C. Wei, J. Martinez, K. Leung, and R. Orwig for their involvement in system development and evaluation.

\section{References}

Anderson, J. R. (1983). The architecture of cognition. Cambridge, MA: Harvard University Press.

Anderson, J. R. (1985). Cognitive psychology and its implications (2nd ed.). New York: W. H. Freeman.

Bechtel, W., \& Abrahamsen, A. (1991). Connectionism and the mind: An introduction to parallel processing in networks. Cambridge, MA: Basil Blackwell.

Carbonell, J. G., Michalski, R. S., \& Mitchell, T. M. (1983). An overview of machine learning. In R. S. Michalski, J. G. Carbonell, \& T. M. Mitchell, (Eds.), Machine learning, an artificial intelligence approach, (pp. 3-23). Palo Alto, CA: Tioga.

Carmel, E.. Crawford, S., \& Chen, H. (1992). Browsing in hypertext: A cognitive study. IEEE I ransactions on Systems, Man and (ybernetics, 22(5), 865-884.

Charniak, E. (1981). A common representation for problem solving and language comprehension information. Artificial Intelligence, 16 , 225-255.

Chen, H., \& Dhar, V. (1987). Reducing indeterminism in consultation: A cognitive model of user/librarian interaction. In Proceedings of the 6th National Conference on Artificial Intelligence (AAAI-87) (pp. 285-289). Seattle, WA: Morgan Kaufmann Publishers.

Chen. H., \& Dhar, V. (1991). Cognitive process as a basis for intelligent retrieval systems design. Information Processing and Management, $27(5), 405-432$.

Chen, H., \& Lynch, K. J. (1992). Automatic construction of networks of concepts characterizing document databases. IEEE Transactions on System., Man and Crbernetics, 22(5), 885-902.

Chen, H., Lynch, K. J., Basu. K., \& Ng. T. (1993). Generating, integrating, and activating thesauri for concept-based document retrieval. IEEE EXPERT, Special Series on Artificial Intelligence in Text-Based Information Systems, 8(2), 25-34.

Chen, H., Schatz, B,, Yim, T., \& Fye, D. (in press). Automatic thesaurus generation for an electronic community system. Journal of the American Society for Information Science.

Cohen, P. R.. \& Kjeldsen, R. (1987). Information retrieval by constrained spreading activation in semantic networks. Information Processing and Management, 23(4), 255-268.

Dalton, J., \& Deshmane, A. (1991). Artificial neural networks. IEEE Potentials, 10(2), 33-36.

Derthick, M. (1988). Mundane reasoning by parallel constraint satisfaction. Unpublished doctoral dissertation, Carnegie Mellon University, Pittsburgh, PA.

Dietterich, T. G., \& Michalski, R. S. (1983). A comparative review of selected methods for learning from examples. In R. S. Michalski, J. G. Carbonell, \& T. M. Mitchell (Eds.), Machine learning, an artificial intelligence approach (pp. 41-81). Palo Alto, CA: Tioga.

Ekmekcioglu, F. C., Robertson, A. M., \& Willett, P. (1992). Effectiveness of query expansion in ranked-output document retrieval systems. Journal of Information Science. 18. 139-147.

Everitt, B. (1980). Cluster analysis (2nd Ed.). London: Heinemann Educational Books.

Fahlman, S. E. (1979). NEIL: A system for representing and using realworld knowledge. Cambridge, MA: MIT Press.

Foss, C. L. (1989). Tools for reading and browsing hypertext. Information Processing and Management, 25(4), 407-418.

Frawley, W. J., Pietetsky-Shapiro, G., \& Matheus, C. J. (1991). Knowledge discovery in databases: An overview. In G. Piatetsky-Shapiro \& W. J. Frawley (Eds.), Knowledge discovery in databases, (pp. 1-30). Cambridge, M^: MIT Press.

Gallant, S. I. (1988). Connectionist expert system. Communications of the $A C M, 3 l(2), 152-169$.

Goodman, S. E., Mehrer, A. L., Lynch, K. J., \& Roche, E. M. (1990). International developments in the information technologies: The Mosaic group at The University of Arizona. In J. F. Nunamaker \& R. H. Sprague (Eds.), Proceedings of the 23rd Annual HICSS Confer- 
ence, decision support and knowledge based systems track, (pp. 605611). Kaui, Hawaii: IEEE Computer Society Press.

Hinton, G. E., \& Sejnowski, T. J. (1986). Learning and relearning in Boltzmann machines. In D. E. Rumelhart, J. L. McClelland, \& the PDP Research Group (Eds.), Parallel distributed processing (pp. 282-317). Cambridge, MA: MIT Press.

Hopfield, J. J. (1982). Neural network and physical systems with collective computational abilities. Proceedings of the National Academy of Science, USA, 79(4), 2554-2558.

Humphreys, B. L., \& Lindberg, D. A. (1989). Building the unified medical language system. In Proceedings of the Thirteenth Annual Symposium on Computer Applications in Medical Care. (pp. 475-480). Washington, DC: IEEE Computer Society Press.

Kaufman, K. A., Michalski, R. S., \& Kerschberg, L. (1991). Mining for knowledge in databases: Goals and general description of the INLEN system. In G. Piatetsky-Shapiro \& W. J. Frawley (Eds.), Knowledge discovery in databases (pp. 449-462). Cambridge, MA: MIT Press.

Knight, K. (1990). Connectionist ideas and algorithms. Communications of the $A C M, 33(11), 59-74$.

Lindberg. D. A., \& Humphreys. B. L. (1990). The UMLS knowledge sources: Tools for building better user interface. In Proceedings of the Fourteenth Annual Symposium on Computer Applications in Medical Care. (pp. 12 l-125). Los Alamitos, CA: Institute of Electrical and Electronics Engineers.

Lippmann, R. P. (1987). An introduction to computing with neural networks. IEEE Acoustics Speech and Signal Processing Magazine. $4(2), 4-22$.

Lynch, K. J., Snyder, J. M., McHenry, W. K., \& Vogel, D. R. (1990). The Arizona Analyst Information System: Supporting collaborative research on international technological trends. In A. Verignstuart (Ed.), Proceedings of the IFIP WG8.4 Conference on Multi-user Interfaces and Applications (pp. 159-174). Crete, Greece: North-Holland.

McCray, A. T., \& Hole, W. T. (1990). The scope and structure of the first version of the UMLS semantic network. In Proccedings of the Fourteenth Annual Symposium on Computer Applications in Medical Care (pp. 126-130). Los Alamitos, CA: Institute of Electrical and Electronics Engineers.

McHenry, W. K., Lynch, K. J., \& Snyder, J. M. (1990). Cross-cultural information tcchnologics research: The case of Eastern Europe and the USSR. In J. I. DeGross, M. Alavi, \& H. Oppelland (Eds.), Proceedings of the Eleventh International Conference on Information Systems. Copenhagen, Denmark: ACM.

Michalski, R. S., \& Larson, J. B. (1978). Selection of most representative training examples and incremental generation of VL1 hypotheses. The underlying methodology and the description of programs ESEL and $A Q 11$. University of Illinois, Department of Computer Science.

Michalski, R. S., \& Stepp, R. E. (1983). Learning from observation: Conceptual clustering. In R. S. Michalski, J. G. Carbonell, \& T. M. Mitchell (Eds.), Machine learning, an artificial intelligence approach, (pp. 331-363). Palo Alto, CA: Tioga.

Monarch, I. \& Carbonell, J. G. (1987). CoalSORT: A knowledge-based interface. IEEE EXPERT, 2(1), 39-53.

Montgomery, D. D. (1976). Design and analysis of experiments. New York: John Wiley \& Sons.
Morrison, D. F. (1976). Multivariate statistical methods. New York: McGraw-Hill.

Nelson, C. R. (1973). Applied time series analysis for managerial forecasting. San Francisco: Holden-Day.

Parsaye, K., Chignell, M., Khoshafian, S., \& Wong, H. (1989). Intelligent databases. New York: John Wiley \& Sons.

Parsaye, K., Chignell, M., Khoshafian, S., \& Wong, H. (1990). Intelligent databases. AI Expert, 5(3), 38-47.

Peat, H. J., \& Willett, P. (1991). The limitations of term co-occurrence data for query expansion in document retrieval systems. Journal of the American Society for Information Science, 42(5), 378-383.

Quillian, J. R. (1983). Learning efficient classification procedures and their application to chess end games. In R. S. Michalski, J. G. Carbonell, \& T. M. Mitchell (Eds.), Machine learning, an artificial intelli- gence approach, (pp. 463-482). Palo Alto, CA: Tioga.

Quillian, M. R. (1968). Semantic memory. In M. Minsky (Ed.), Semantic information processing. (pp. 227-270). Cambridge, MA MIT Press.

Rich, E., \& Knight, K. (1991). Artificial intelligence (2nd ed.). New York: McGraw-Hill.

Rumelhart, D. E., Hinton, G. E., \& Williams, R. J. (1986). Learning internal representations by error propagation. In D. E. Rumelhart, J. L. McClelland, \& the PDP Research Group (Eds.), Parallel distributed processing, (pp. 318-362). Cambridge, MA: MIT Press.

Ryan, B. F., Joiner, B. I., \& Ryan, T. A. (1985). MINITAB handhook (2nd ed.). Boston: PWS-KENT.

Salton, G. (1989). Automatic text processing. Reading, MA: AddisonWesley.

Salton, G., Allan, J., \& Buckley, C. (1994). Automatic structuring and retrieval of large text files. Communications of the $A C M, 37(2)$, 97-108.

Shastri, L. (1991). Why semantic networks? In J. F. Sowa (Ed.), Principles of semantic networks: Explorations in the representation of knowledge, (pp. 109-136). San Mateo, CA: Morgan Kauffmann.

Shoval, P. (1985). Principles, procedures and rules in an expert system for information retrieval. Information Processing and Management, $21(6), 475-487$.

Simpson, P. K. (1990). Artificial neural systems: Foundations, paradigms, applications, and implementations. New York: McGraw-Hill.

Sowa, J. F. (1991a). Issues in knowledge representation. In J. F. Sowa (Ed.), Principles of semantic networks: Explorations in the representation of knowledge, (pp. 1-12). San Mateo, CA: Morgan Kauffmann.

Sowa, J. F. (1991b). Panel: Current issues in semantic networks. In J. F. Sowa (Ed.), Principles of semantic networks: Explorations in the representation of knowledge, (pp. 13-43). San Mateo, CA: Morgan Kauffmann.

Sparck Jones, K. (1971). Automatic keyword classification for information retrieval. London: Butterworths.

Tank, D. W., \& Hopfield, J. J. (1987). Collective computation in neuronlike circuits. Scientific American, 257(6), 104-1 14.

Touretzky, D., \& Hinton, G. E. (1988). A distributed connectionist production system. Cognitive Science, 12(3), 423-466.

Winston, P. H. (1984). Artificial intelligence (2nd ed.). Reading, MA: Addison-Wesley. 